\title{
Raman Spectroscopy for Characterizing Porous Carbon Material
}

Rima Zuriah Amdani

Thesis submitted for the degree of Master in Science

Project duration: 9 months

Supervised by Christian Brackmann and Frederik Ossler

Department of Physics

Division of Combustion Physics

May 2020 


\section{Abstract}

Porous carbon obtained from low cost and abundant biomass has gained attention as renewable material feedstock. In this study, porous carbon biochar produced through pyrolysis was investigated by Raman spectroscopy to disclose the structural properties. The measured samples were in form of pellets (identified as WP, 139, 233, 226), and lumber wood (Pine, a softwood type and Beech, a hardwood type). The resulted spectra, which consisted of two broadened overlapped peaks were fitted with two Gaussian curves. The intensity ratio of the curves (ID/IG) were calculated to analyse the aromaticity of the samples. The aromaticity represents the proportion of the total $\mathrm{C}$ phases (amorphous and crystalline) within the samples. The result indicates that the aromaticity of the samples increased as the pyrolysis temperature was raised. Furthermore, the biochar obtained for higher temperature had larger crystalline surface area, which is indicated by $\mathrm{L}_{a}$ (crystalline length). The $\mathrm{L}_{\mathrm{a}}$ values obtained were around 9-10 $\AA$ and exhibit a highly disordered structure with turbostratic arrangement. As complementary, the data from Raman measurements were also compared with elemental analysis results to show the amount of carbon content in the samples. The results from elemental analysis present that the higher temperature will produce higher carbon proportion in the samples. Thereby, both Raman spectroscopy and elemental analysis give similar trends for evaluating the structure of the samples. In comparison with a soot sample, biochar exhibits less carbonization since the aromaticity and crystalline surface area (defined by $\mathrm{L}_{\mathrm{a}}$ ) of the soot have higher values. The Raman signal increases for higher laser power and detector integration acquisition time. However, high power and long acquisition time promote fluorescence background. Thus, moderate laser power and integration time was preferably chosen for reliable Raman measurements. 


\title{
Acronyms and Abbreviation
}

\author{
CHP Combined Heating Power \\ XRD X-Ray Diffraction \\ HRTEM High Resolution Transmission Microscopy \\ SEM Scanning Electron Microscope \\ GC Gas Chromatography \\ NMR Nuclear Magnetic Resonance \\ ESR Electron Spectroscopy Resonance \\ ESCA Electron Spectroscopy for Chemical Analysis \\ PES Photoelectron Spectroscopy \\ HTT Heating Treatment Temperature \\ GoF Goodness of Fit \\ SNR Signal-to-Noise
}




\section{Contents}

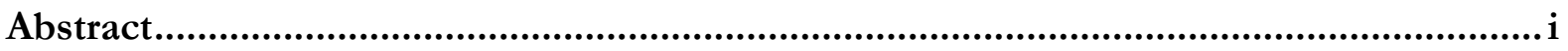

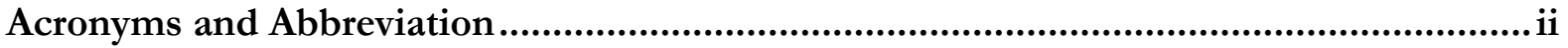

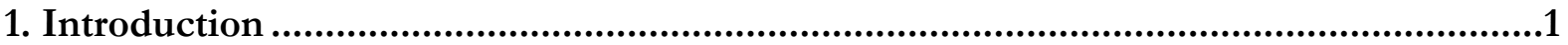

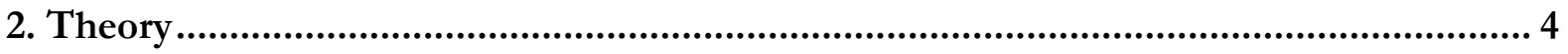

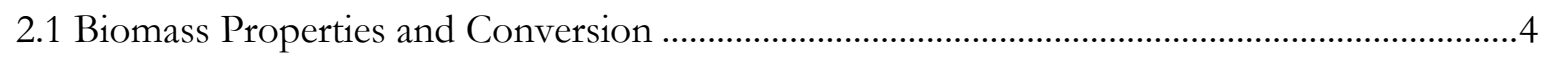

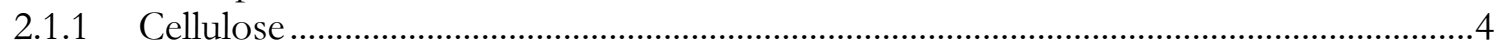

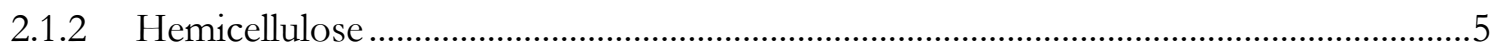

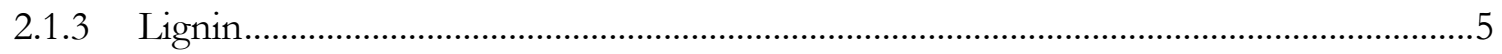

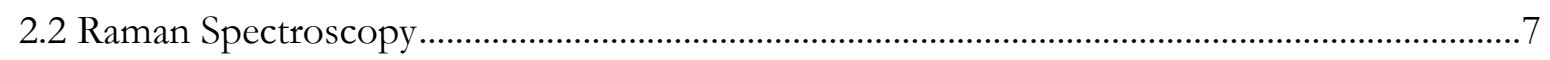

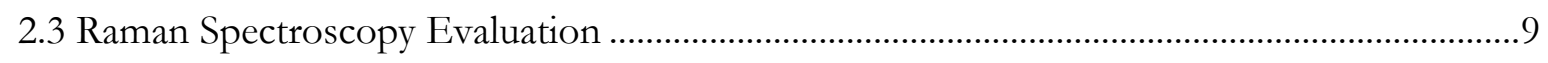

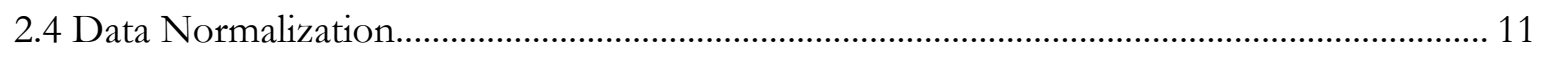

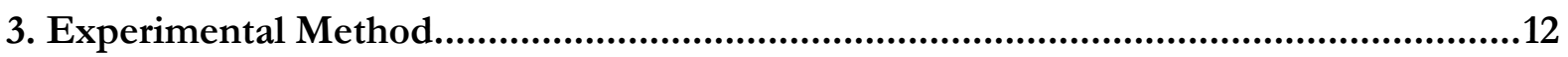

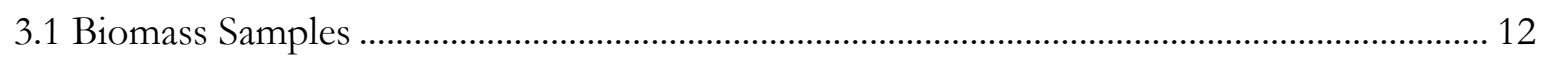

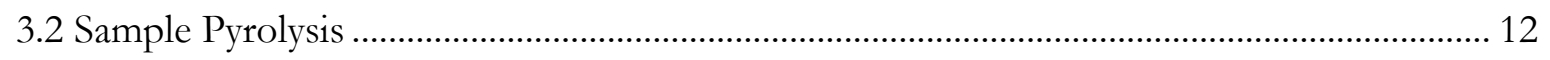

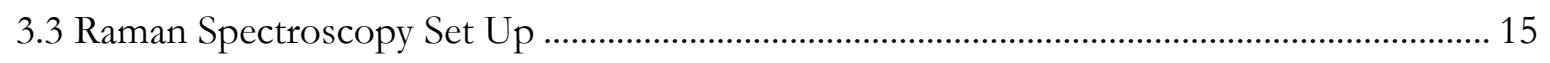

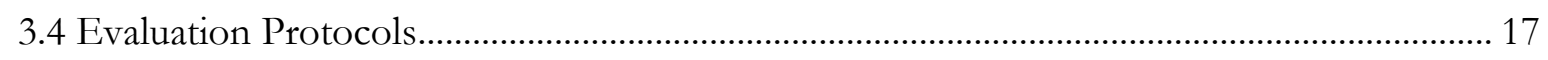

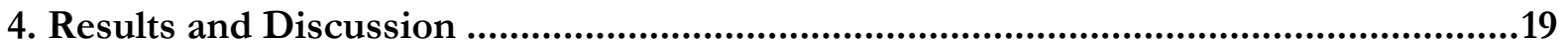

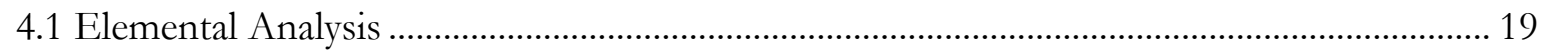

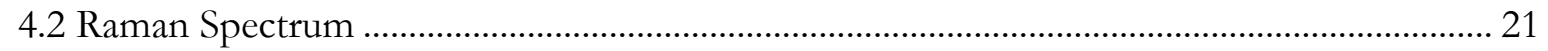

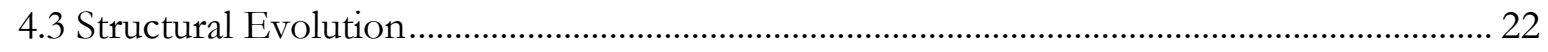

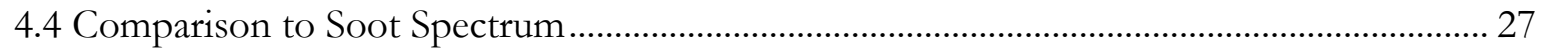

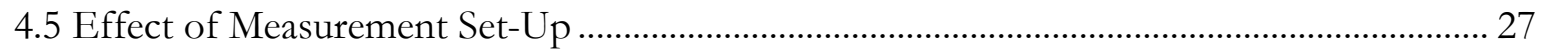

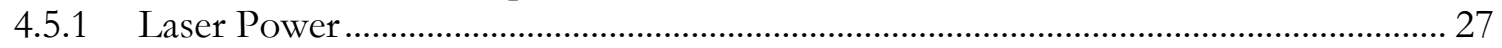

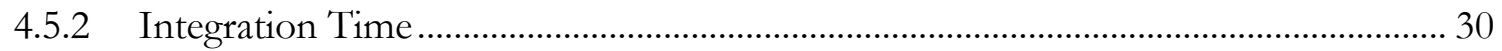

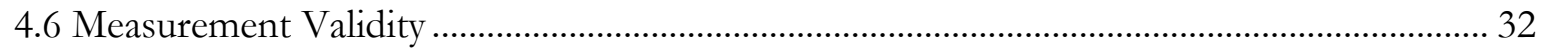

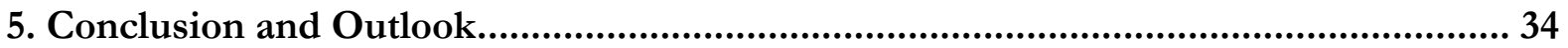

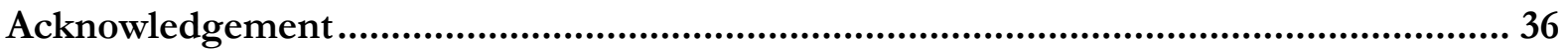




\section{Chapter 1}

\section{Introduction}

Presently, the concept of sustainability draws more attention in a variety of aspects of human life. This issue leads to the primary use of renewable resources into many applications including in the industrial sector. Referring to that, porous carbon, which can be obtained from low cost and abundant biomass have gained high interest as a starting material. Although it also yields a shortage such as lower heating value and bulk density, some procedures such as pretreatment and preheating are continuously developed to enhance the capability.

Biomass utilization exhibits the transition towards environmentally friendly technology. It has been known as contributor in soil fertility and water treatment [1]. Due to the oxidation, the polycyclic aromatic structure of porous carbon will enable to bind carbocyclic groups, which can increase the capability to store the nutrient in the soil. Moreover, porous carbon is chemically and microbially stable, which can keep the fertility in the soil to last longer [2].

As for energy supplies, biomass can be utilized as biofuel in power plants to generate electricity, heat, and to produce vehicle fuels for daily transportation. In Sweden, around $85 \%$ of the bioenergy source is obtained from biomass forest, which is dominated by two species (Scots pine and Norway spruce) [3]. Mostly, the biopower is produced in form of combined heating and power $(\mathrm{CHP})$ installation in forest-based industries and in district heating. For example, Ingelsta CHP is the largest CHP supplied by wood chip in Sweden as illustrated in Figure 1.1 [4]. Furthermore, development of biomass utilization has an indirect role in climate change due to the reduction of greenhouse gas $\left(\mathrm{CO}_{2}\right)$ in the atmosphere [5].

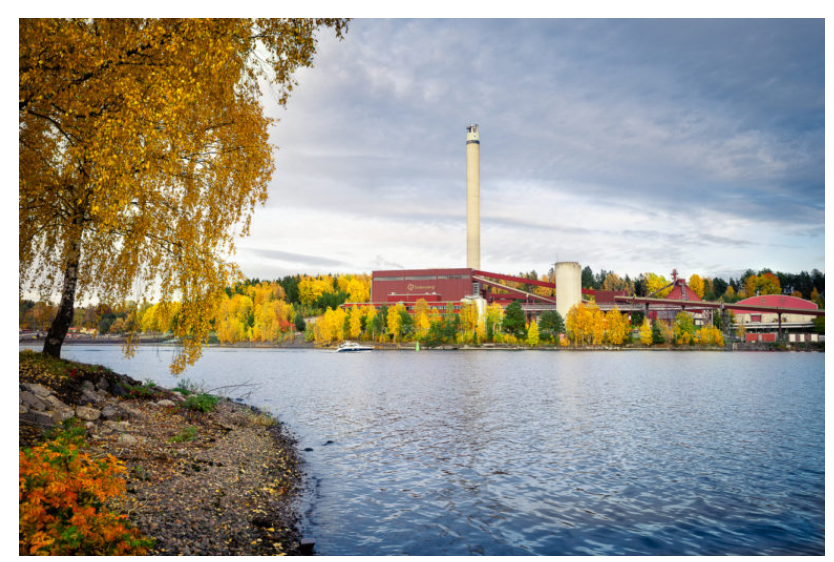

Figure 1.1 The Ingelsta CHP located in Södertalje, south of Stockholm [6]. 
In industrial bio-based products, porous carbon has potential ability as material for capacitors, electrodes, and catalyst, as absorbents, and for gas storage [7,8]. Figure 1.2 illustrates the process for production of a supercapacitor from biomass-derived porous carbon. This material exhibits a large surface area of micropores, promising high specific capacitance. However, the deficiency of mesopores and macropores affect the performance such as cycling stability [9]. In order to improve the limitation, synthesis of hierarchical porous carbon is compulsory and should be developed to get the best results.

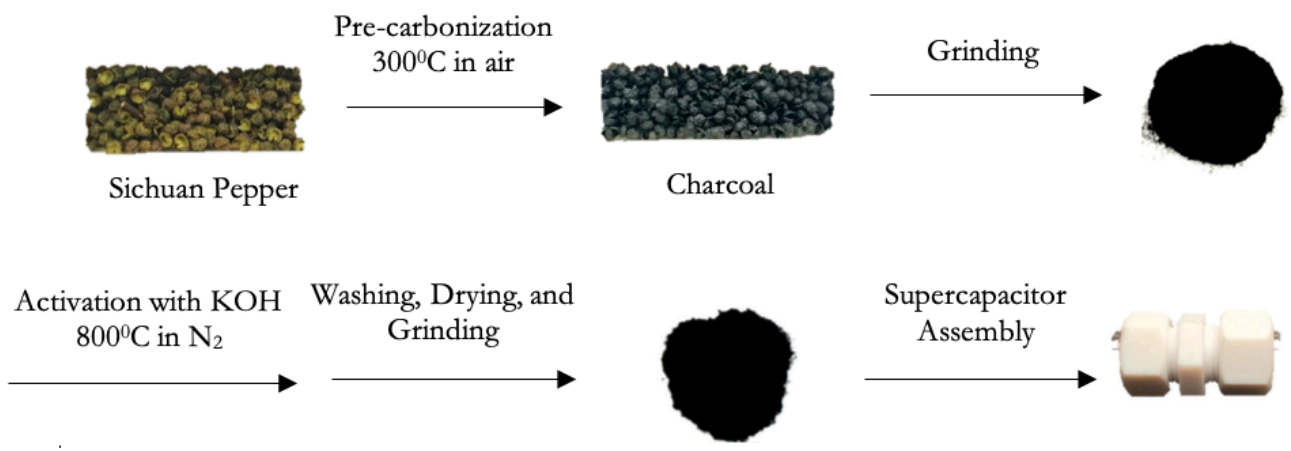

Figure 1.2 Application of porous carbon as supercapacitor [9].

Generally, production of porous carbon is mainly through pyrolysis and activation of organic carbonaceous materials i.e., biomass [7,10]. These process can change the structural and chemical properties of the substance. The pyrolysis results in a biomass char (biochar), which is physically and chemically different from its origin. The heterogeneity and complexity of the char structure remains a challenge to identify. Meanwhile, the selection and treatment of porous carbon for specific purposes depends on its characteristics. Thus, the necessity for characterization of the charred material and its evolution requires suitable methods to get adequate information.

Investigation of the material structure can be done by multiple spectroscopy and imaging techniques. X-Ray Diffraction (XRD), High Resolution Transmission Microscopy (HRTEM), and Scanning Electron Microscopy (SEM) are frequently utilized for analyzing the morphology of ordered carbon. The biochars are highly disordered porous carbon materials, which has less polycrystalline structure with defect [11]. Other methods such as Gas Chromatography (GC) and Nuclear Magnetic Resonance (NMR) provide information on the chemical composition but require extensive sample preparation and data analysis [12]. Raman spectroscopy is based on inelastic scattering of light and depends on the rotational and vibrational energy in a molecule. Since this is an optical measurement, it is non-intrusive to the sample. Moreover, it is sensitive to measure the disordered structure of biochars. The Raman techniques allows to examine the amorphous structure of porous carbon with less sample preparation and analysis time because the 
measurement can be performed directly to the samples [13]. Thus, the structural properties of any porous carbon can be effectively analyzed by this spectroscopy technique.

Numerous experiments with Raman spectroscopy have been carried out. It proves to be a suitable result for disclosing structural properties from several materials [14-17]. In a recent study, carbonaceous samples such as soot and carbon blacks have been characterized using this method [18]. Another study with Raman spectroscopy presents that heat treatment of the sample increases the degree of carbonization [19]. In this study, measurements will be performed to characterize solid porous carbon from biomass that undergo pyrolysis. The result is expected to give insights about the characteristics of the material evolution, especially on the aromatic structure ring systems. Furthermore, the factors that affect the measurements will be presented to have better understanding for the research. As complementary information, the results from elemental analysis are finally compared with the finding from Raman measurement to obtain a thorough investigation of the samples. 


\section{Chapter 2}

\section{Theory}

\subsection{Biomass Properties and Conversion}

Biomass is defined as the biodegradable fraction of products, waste and residues with a biological origin (vegetal or animal substance) ranging from agriculture, forestry, fisheries, aquaculture, and other related industry [10]. The agricultural residues such as wood-like or grass are commonly categorized as lignocellulosic biomass. They are mostly comprised of cellulose, hemicellulose, lignin, extractives, and inorganic substances (ash) [20]. The composition of some biomass species is presented in Table 2.1 [5].

Table 2.1 Composition of biomass species

\begin{tabular}{|c|c|c|c|c|c|}
\hline Species & Cellulose & Hemicellulose & Lignin & Extractives & Ash \\
\hline Softwood (average) $^{1}$ & 45.8 & 24.4 & 28.0 & n.a & 1.7 \\
\hline Hardwood (average) $^{1}$ & 45.2 & 31.3 & 21.7 & n.a & 2.7 \\
\hline Corn stalk ${ }^{2}$ & 42.7 & 23.6 & 17.5 & 9.8 & 6.8 \\
\hline Rice straw ${ }^{2}$ & 37.0 & 22.7 & 2.6 & 2.1 & 16-19 \\
\hline Olive husk $^{1}$ & 24.0 & 23.6 & 48.4 & n.a & 4.0 \\
\hline
\end{tabular}

\subsubsection{Cellulose}

Based on table 2.1, the softwood and hardwood types have a similar amount of cellulose, while the others contain less cellulose. As illustrated in Figure 2.1, cellulose is a homopolysaccharide group consisting of $\beta(1 \rightarrow 4)$ linked with D-glucose $\left(\mathrm{C}_{6} \mathrm{H}_{10} \mathrm{O}_{5}\right)_{\mathrm{n}}$ units. It is the most combustible part, followed by lignin. The hydroxyl $(\mathrm{O}-\mathrm{H})$ groups tend to hold oxygen in other chains to form microfibril with strong tensile strength and a higher amount of crystallinity than the amorphous structure. The hydrogen-bond in cellulose is not easily dissolved with a solvent, but it can be broken through a heating process [20].

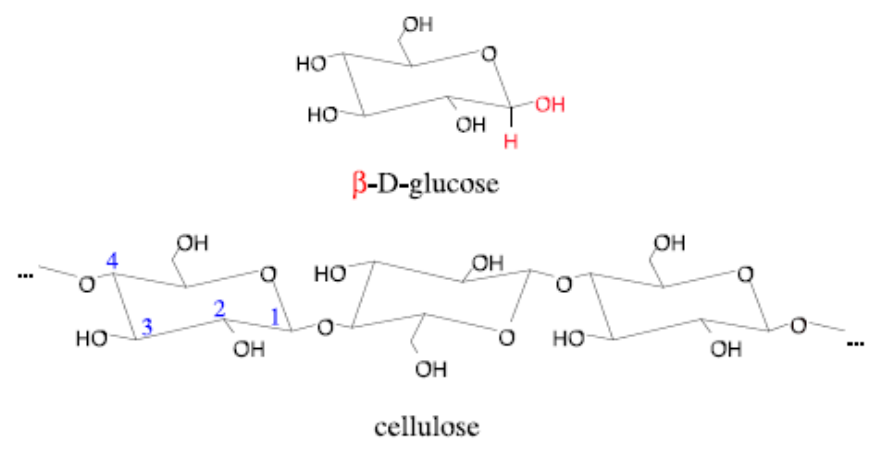

Figure 2.1 Cellulose structure [20]. 


\subsubsection{Hemicellulose}

In contrast to cellulose, hemicellulose is made from heteropolysaccharides derived from sugar. It contains a large amount of random amorphous structure with less mechanical strength [5]. Thus, it is easier to hydrolyze and can be dissolved in a solution. The bonding is believed from adhesion properties of the molecule itself [20]. Table 2.1 explains that the hardwood has a higher content of hemicellulose than softwood, while the others present a similar amount of it.

\subsubsection{Lignin}

In comparison with hardwood, softwood contains more lignin. Lignin is a complex chemical compound of polymers phenylpropane units. It consists of groups from phenolic hydroxyl, aldehyde in the side chain, and methoxy, which are shown in Figure 2.2. The connection between phenylpropane units can be linked with $\mathrm{C}-\mathrm{O}-\mathrm{C}$ or $\mathrm{C}-\mathrm{C}$ bonds [5]. Lignin occupies space between cellulose and hemicellulose in the cell wall as presented in Figure 2.3. It is crosslinked with cellulose and covalently linked with hemicellulose. The distribution and presence of lignin can be altered by some pretreatment procedures [20].

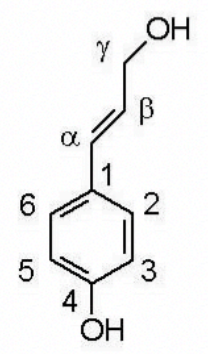

p-coumaryl alcohol<smiles>COc1cc(/C=C/CO)ccc1O</smiles>

Coniferyl alcohol<smiles>COc1cc(/C=C/CO)cc(OC)c1O</smiles>

Sinapyl alcohol

Figure 2.2 Lignin monomers [20].

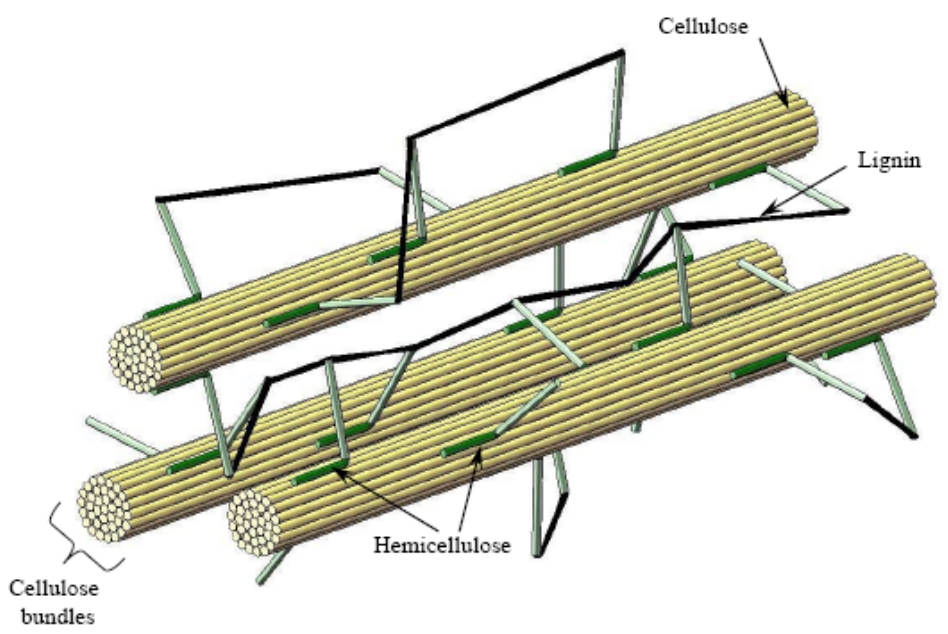

Figure 2.3 Distribution of cellulose, hemicellulose, and lignin in the cell wall [20]. 
Biomass exhibits several drawbacks compared to fossil materials such as a lower thermal content, higher moisture, and lower bulk density. Hence, biomass is usually changed to other forms to enhance the energy density. The aim of the conversion process is to release the water content, which can improve the thermal value and dense the form of the materials. Generally, the conversion methods are classified into biological and thermal processes. Hydrolysis and fermentation are some examples of biological types, while the thermal processes involve pyrolysis, gasification, and liquefaction [5].

Pyrolysis is the heating process of solid material in an inert atmosphere. The products from pyrolysis of biomass can be in the form of gaseous $\left(\mathrm{CO}_{2}, \mathrm{H}_{2}, \mathrm{CO}, \mathrm{C}_{2} \mathrm{H}_{2}\right.$, alkene $\left(\mathrm{C}_{2} \mathrm{H}_{4}\right)$, alkane $\left(\mathrm{CH}_{4}, \mathrm{C}_{2} \mathrm{H}_{6}\right)$, benzene, and tar), liquid (water, tar), and solid (char). The main component of char is primarily carbon, and it is affected by the feedstock type and pyrolysis condition such as temperature, residence time, and heating rate [21]. The pyrolysis of biomass, which is made at slow heating rate has the purpose to maximize the production of the biochar [5]. Increasing the pyrolysis temperature does not transform the biochar into graphite form but influences the aromaticity. An illustration of the biochar structure is shown in Figure 2.4 that consists of a carbonized phase and noncarbonized phase. The carbonized phase exhibits two different aromatic $\mathrm{C}$ phases (amorphous and crystalline phases). The aromatic rings in the amorphous phase are randomly oriented, while in crystalline phase condensed polyaromatic sheets are likely turbostratically arranged. The sheets in a turbostratic arrangement are not perfectly stacked and aligned, such as in graphite. The aromaticity describes the proportion of both aromatic $\mathrm{C}$ phases in the material. Meanwhile, the degree of aromatic condensation examines the condensed phase only and is more difficult to evaluate [22].

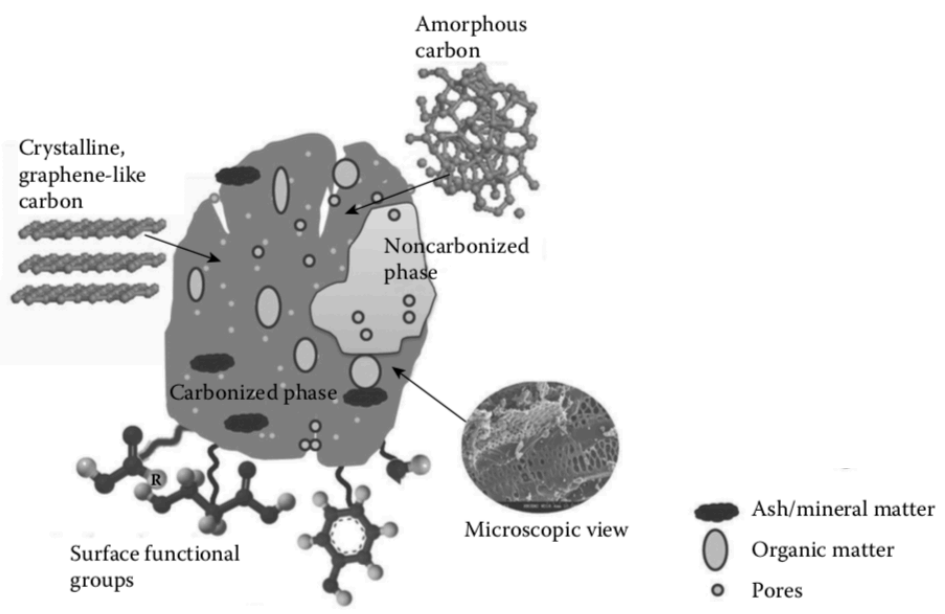

Figure 2.4 Biochar structure [21]. 


\subsection{Raman Spectroscopy}

Spectroscopy is related to the interaction of an electromagnetic wave with a matter. By this mean, spectroscopy can provide information about the structure of matter. Meanwhile, it is notably known that energy is quantized. A transition from one energy level to another in a molecule will require a specific amount of energy rather than an arbitrary. The electromagnetic wave region contains the allowed types of transition of the molecule, as presented in Table 2.2 [23]. As an example, microwave radiation can give rise to the transition between rotational energy levels in the molecule.

Table 2.2 Corresponding type of transition of each wavenumber range in the electromagnetic spectrum

\begin{tabular}{|c|c|c|c|c|}
\hline \multirow{3}{*}{ Radiofrequency } & $\left.m^{-1}\right)$ & Spectroscopy & Transition & \multirow{3}{*}{$\begin{array}{l}\text { Change of } \\
\text { spin }\end{array}$} \\
\hline & $10^{-4}-10^{-2}$ & $\begin{array}{l}\text { Nuclear magnetic } \\
\text { resonance (n.m.r) }\end{array}$ & $\begin{array}{l}\text { Transition between nuclear } \\
\text { spin level in magnetic field }\end{array}$ & \\
\hline & $10^{-2}-1$ & $\begin{array}{l}\text { Electron spin } \\
\text { resonance (e.s.r) }\end{array}$ & $\begin{array}{l}\text { Transition between electronic } \\
\text { spin level in magnetic field }\end{array}$ & \\
\hline Microwave & $1-10^{2}$ & Rotational & $\begin{array}{l}\text { Transition between rotational } \\
\text { energy level }\end{array}$ & $\begin{array}{l}\text { Change } \\
\text { orientation }\end{array}$ \\
\hline Infra-red & $10^{2}-10^{4}$ & Vibrational & $\begin{array}{l}\text { Transition between vibrational } \\
\text { energy level }\end{array}$ & $\begin{array}{l}\text { Change } \\
\text { Configuration }\end{array}$ \\
\hline Visible-UV & $10^{4}-10^{6}$ & Electronic & $\begin{array}{l}\text { Transition between energy } \\
\text { level of valence electron of } \\
\text { atom and molecule }\end{array}$ & \multirow{2}{*}{$\begin{array}{l}\text { Change of } \\
\text { electron } \\
\text { distribution }\end{array}$} \\
\hline X-Ray & $10^{6}-10^{8}$ & ESCA, PES & $\begin{array}{l}\text { Transition between energy } \\
\text { level of inner electron of atom } \\
\text { and molecule }\end{array}$ & \\
\hline$\gamma$-Ray & $10^{8}-10^{10}$ & & $\begin{array}{l}\text { Rearrangement of elementary } \\
\text { particles in the nucleus }\end{array}$ & $\begin{array}{l}\text { Change of } \\
\text { nuclear } \\
\text { configuration }\end{array}$ \\
\hline
\end{tabular}

Raman spectroscopy is a method that enables the same type of information as obtained with radiation in microwave and infrared range [24]. The Raman technique is based on inelastic scattering process. Initially, radiation of an electromagnetic wave that passes through a substance will be scattered. This phenomenon yields the same as the initial wavelength of light (elastic) or conversely have the wavelength shifted (inelastic), which are known as Rayleigh and Raman scattering, respectively. This frequency shift corresponds to the transition energy of the molecule and describes a fingerprint of molecular bonds. Thus, the Raman spectroscopy can probe vibrational, including the rotational energy in the molecule regardless of the wavelength origin.

In Raman scattering, the photon energy that passes on to the molecule causes it to be excited into a virtual state that rises the molecule vibration, rotation, or both of them. Then, it will deexcite to 
a level of either higher or lower energy than the initial one. A scattered photon of either higher or lower energy than the incoming photon is known as Stokes and anti-Stokes scattering sequentially [25]. An illustration of the transition energy in Raman scattering is presented in Figure 2.5. Basically, the generation of an anti-Stokes process is more difficult to achieve than the Stokes in a room temperature since it needs to start in a higher energy than the ground state to allow the transition to a state lower than the initial state.

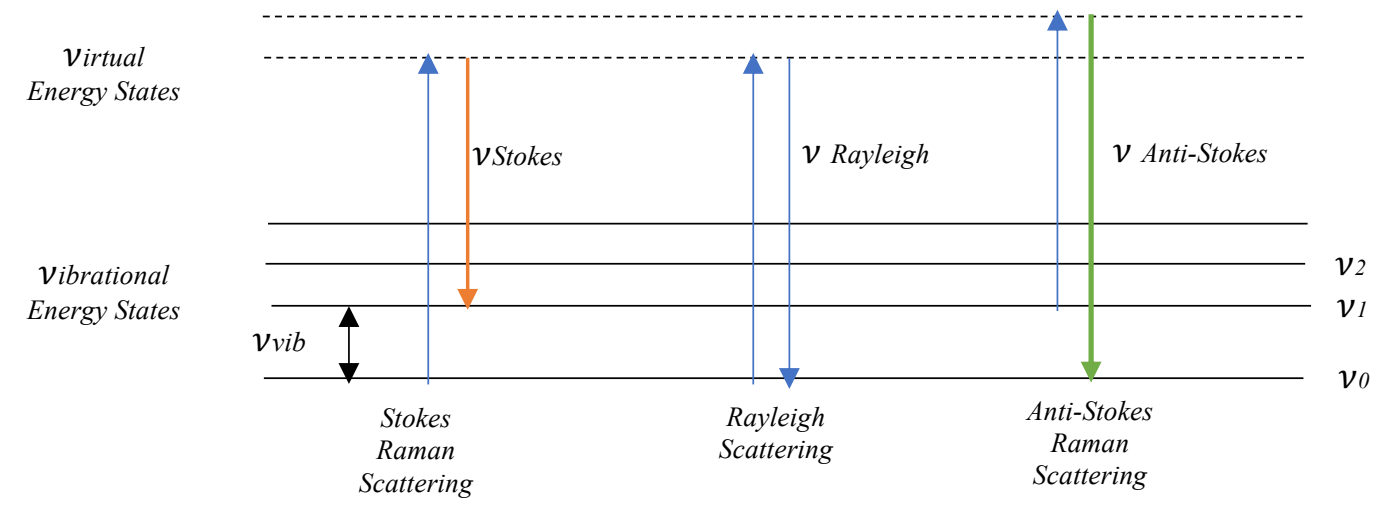

Figure 2.5 Raman scattering schematic energy level diagram.

The activation of the Raman effect is based on molecular polarizability $(\alpha)$. A molecule that is placed in an electric field $(E)$, such as from laser light, will experience a distortion. The negative charge is attracted to a positive pole and inverse for the positive charged nuclei. This separation of the charge gives an induced electric dipole moment $(P)$ and has defined in Equation (2.1). Hence, the ability of the molecule to produce an induced dipole moment depends on the magnitude of electric field and the ease by which the molecule can be distorted (polarizability, $\alpha$ ) [24].

$$
P=\alpha . E
$$

The function of an oscillating electric field is given by Equation (2.2). The electric field strength $(E)$ of an electromagnetic wave (laser light) fluctuates with the time $(t) . E_{0}$ represents the vibrational amplitude, and $v_{l}$ is the laser frequency [24].

$$
E=E_{0} \sin 2 \pi v_{l} t
$$

The polarizability of the molecule will change periodically due to the internal motion (vibrational or rotational). Thus, the induced dipole moment is superimposed upon these motions. Consider for the vibrational case, the function of the molecule polarizability that is altered by the vibration frequency follows in Equation (2.3). $\alpha_{0}$ is the equilibrium polarizability, and $\beta$ defines the rate of change in polarizability with the vibration [24]. 


$$
\alpha=\alpha_{0}+\beta \sin 2 \pi v t
$$

Inserting Equation (2.2) and (2.3) into (2.1) and rearranging by the trigonometric function will result in Equation (2.4)

$$
\begin{gathered}
P=\left(\alpha_{0}+\beta \sin 2 \pi v_{v i b} t\right)\left(E_{0} \sin 2 \pi v_{l} t\right) \\
\sin A \sin B=\frac{1}{2}[\cos (A-B)-\cos (A+B)] \\
P=\frac{\alpha_{0} E_{0} \sin 2 \pi v_{l} t}{\text { Rayleigh }}+\frac{1}{2} \beta E_{0} \frac{\left[\cos 2 \pi\left(v_{l}-v_{v i b}\right)\right.}{\text { Stokes }}-\frac{\left.\cos 2 \pi\left(v_{l}+v_{v i b}\right)\right]}{\text { Anti-Stokes }}
\end{gathered}
$$

If the motion (in this case vibration) does not change the polarizability tensor $(\beta=0)$, then it only generates the elastic Rayleigh scattering [24]. The polarizability of the molecule depends on the symmetry of the molecule. Therefore, the activation of the vibrational mode to acquire a Raman spectrum will rely on the molecule symmetry. Carbon functional groups such as $\mathrm{C}-\mathrm{C}, \mathrm{C}=\mathrm{C}, \mathrm{C} \equiv \mathrm{C}$, $\mathrm{C}-\mathrm{H}$, and $\mathrm{H}-\mathrm{C}-\mathrm{H}$ highly change the polarizability and can produce strong Raman signals [26].

Generally, the power of the recorded Raman signal $\left(P_{r}\right)$ is proportional to the incident laser power source $\left(P_{i}\right)$ as expressed in Equation (2.5). In this formula, the signal strength is also affected by several factors such as number density of the scattering species (n), different Raman cross-sections $\left(\frac{\partial \sigma}{\partial \Omega}\right)$, the solid detection angle $(\Omega)$, and the sampling volume extent $(\ell)$. Furthermore, the detection apparatus gives a contribution to collection efficiency $(\epsilon)$. Compared to other measurement techniques such as Mie scattering, Rayleigh scattering, and laser induced fluorescence (LIF), Raman scattering produces a very weak signal [25].

$$
P_{r}=P_{i} n\left(\frac{\partial \sigma}{\partial \Omega}\right) \Omega \ell \epsilon
$$

\subsection{Raman Spectroscopy Evaluation}

The features of Raman spectra from highly ordered graphite to amorphous carbon material are presented in Figure 2.2. In brief, the Raman spectrum of the carbon material is divided into the first and second order region. The main peaks in the first order region $\left(1100-1800 \mathrm{~cm}^{-1}\right)$ are termed as the $D$ and $G$ peaks, which appear around $1380 \mathrm{~cm}^{-1}$ and $1580 \mathrm{~cm}^{-1}$ respectively. The $G$ peak originates from the bond stretching of $\mathrm{sp}^{2}$ in both rings and chains. The $\mathrm{D}$ peak is attributed to the $\mathrm{sp}^{2}$ breathing modes in the rings [27,28]. The overtone or combination of the first order involves two phonon processes that give rise to a peak in the range around $2200-3400 \mathrm{~cm}^{-1}$. In the higher region above $3500 \mathrm{~cm}^{-1}$, the contamination of the polyaromatic hydrocarbons cluster (PAHs) in the sample will raise the fluorescence background and affect the Raman measurement [29]. 


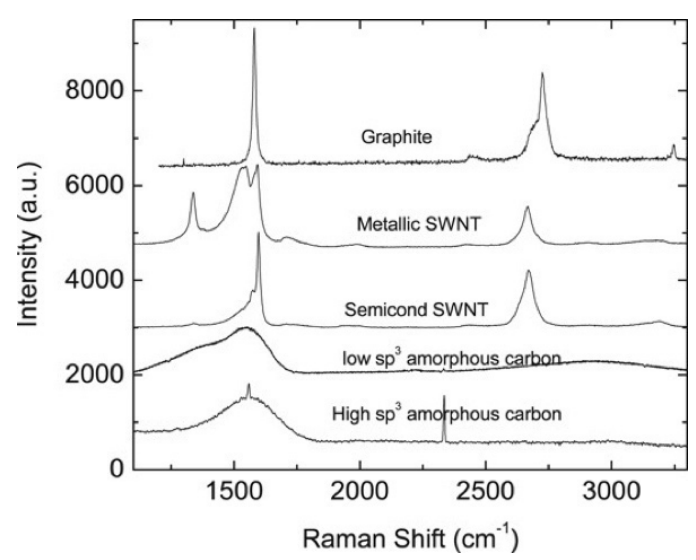

Figure 2. 6 Raman spectra of carbon material [27].

The evaluation of carbon Raman spectra is mainly based on empirical analysis rather than a theoretical study. The correlation between the crystalline length or in-plane size $\left(\mathrm{L}_{2}\right)$ and the intensity ratio of the Raman $G$ and D band was initially presented by Tuinstra and Koenig for analyzing graphite structure. Then, Knight and White derived the formula with a correction factor for a $514.5 \mathrm{~nm}$ laser source as presented in Equation (2.6) [29]. In this project, a coefficient amendment due to a different excitation wavelength has been considered.

$$
\frac{I_{D}}{I_{G}}=\frac{4.4}{L_{a}[\mathrm{~nm}]}
$$

A further study by Ferrari [27] adjusts the proportionality between $L_{a}$ and the relative ratio for disordered structure. It was found that for $\mathrm{L}_{\mathrm{a}}<2 \mathrm{~nm}$, the relation corresponded to $\mathrm{L}_{\mathrm{a}}{ }^{2}$ as illustrated in Figure 2.7. Thus, applying continuity at $2 \mathrm{~nm}$, the new coefficient for the disordered structure is given by Equation (2.7). Based on the research, the $G$ peak position is elevated for disordered carbon as the excitation wavelength decreases from IR to UV. The G peak is dispersed more in disordered structures than in ordered structures such as graphite, nanocrystalline, and glassy carbon. The $\mathrm{D}$ peak always varies with excitation source for all carbon types. However, the $\mathrm{D}$ peak shows less dispersion for disordered structure in contrast to the G peak [27].

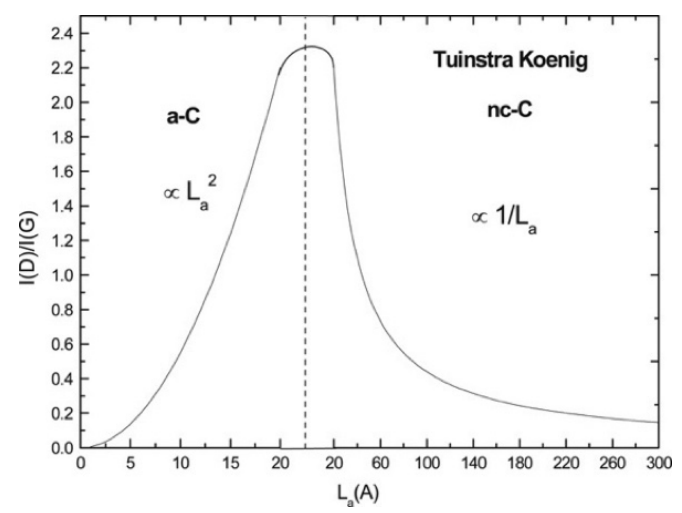

Figure 2.7 Relative intensity ratio dependence for $\mathrm{L}_{\mathrm{a}}[27]$. 


$$
\frac{I_{D}}{I_{G}}=0.0055\left(L_{a}[\AA]\right)^{2}
$$

As already mentioned, the excitation energy can change the coefficient factor used to obtain a more precise $\mathrm{L}_{\mathrm{a}}$ parameter with a different laser source. Equation (2.6) and (2.7) are applicable for $514.5 \mathrm{~nm}$ wavelength. Thus, the suggested formula that complements the previous study is expressed in Equation (2.8). The $514.5 \mathrm{~nm}$ source wavelength is equivalent to $2.41 \mathrm{eV}$. Apply the same continuity at $L_{a}=2 \mathrm{~nm}$, the new correlation to evaluate Raman spectra with $532 \mathrm{~nm}$ laser source is derived in Equation (2.9) [18].

$$
\begin{aligned}
& \frac{I_{D}}{I_{G}}=\frac{4.4}{L_{a}[\mathrm{~nm}]}\left(\frac{2.41}{E_{L}[e V]}\right)^{4} \\
& \frac{I_{D}}{I_{G}}=0.0063(\mathrm{La}[\AA])^{2}
\end{aligned}
$$

Although the $\mathrm{L}_{\mathrm{a}}$ parameter is measured by $\mathrm{XRD}$ or electron microscopy techniques, those methods are limited to the ordered structures. In addition, many studies are conducted to develop Raman spectral analysis. Thus, the Raman method can give prominent result to evaluate the porosity in the carbonaceous material ranging from ordered to the disordered structure through the spectral indicator $\mathrm{L}_{\mathrm{a}}$.

\subsection{Data Normalization}

From statistical standpoint, normalization can have a wide range of meaning. The simple case of normalization is regarded as adjusting the various scale of measured values into the same projection. Thus, the various measurement values $x_{M}$ from different order, unit, or even method can be compared since they have the same standard. Generally, it is transformed into a scale between 0 and 1 by using Equation (2.10) and (2.11) [22]. By these formulae, the measured data depict no unit, and it is commonly denoted as an arbitrary unit (arb. unit).

$$
\begin{gathered}
X_{\text {new }[0-1] \text { or }[1-0]}=\frac{x_{M}-x_{\min }}{x_{\max }-x_{\min }} \\
X_{\text {new }[0-1]}=1-X_{\text {new }[1-0]}
\end{gathered}
$$




\section{Chapter 3}

\section{Experimental Method}

\subsection{Biomass Samples}

The specifics of six biomass samples in the form of pellet and lumber wood are listed in Table 3.1. Some of them are from Vattenfall Research and Development. The others were prepared in the laboratory of Combustion Physics, Lund University. The images of the samples before pyrolysis are presented in Figure 3.1. The samples, which look to having similar size, actually have a different scale. The wood samples are larger since they will shrink during the pyrolysis process.

Table 3.1 List of measured samples and properties (before pyrolysis)

\begin{tabular}{clcc}
\hline No & Identifier & Supplier & $\begin{array}{c}\text { Estimated Dimension } \\
\left(\mathrm{cm}^{3}\right)\end{array}$ \\
\hline a. & WP & - & 0.42 \\
\hline b. & 139 & Vattenfall & 0.77 \\
\hline c. & 233 & Vattenfall & 1.00 \\
\hline d. & 266 & Vattenfall & 0.57 \\
\hline e. & Beech & - & 2.12 \\
\hline f. & Pine & - & 2.04 \\
\hline
\end{tabular}

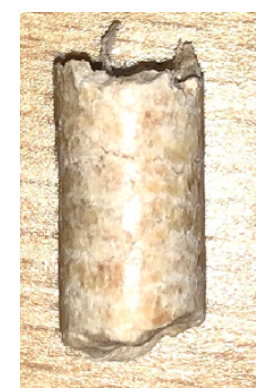

(a)

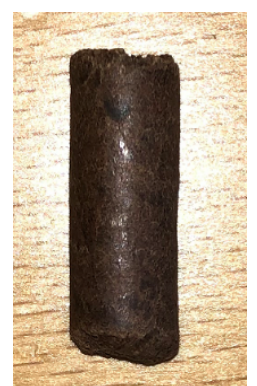

(b)



(c)

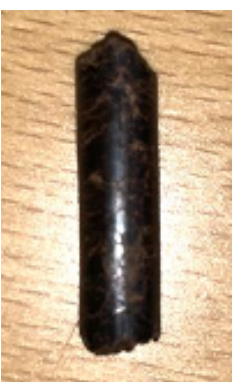

(d)



(e)

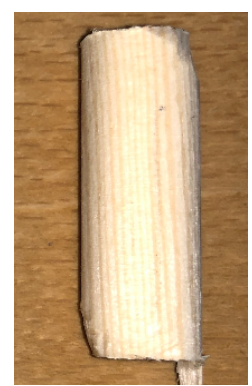

(f)

Figure 3.1 Images of measured samples with the identification as given in Table 3.1.

\subsection{Sample Pyrolysis}

In order to convert the fresh biomass into biochar with a porous carbon structure, the samples were pyrolyzed under various temperatures. Furthermore, the fluorescence background, which can cover the Raman signal, will be reduced. The final temperature of the pyrolysis is varied to know the structural evolution of the samples due to different heat treatments. The pyrolysis was performed in a heating coil, and the temperature is measured using a thermocouple (OMEGA HH802U). The set-up of the pyrolysis equipment is shown in Figure 3.2. 


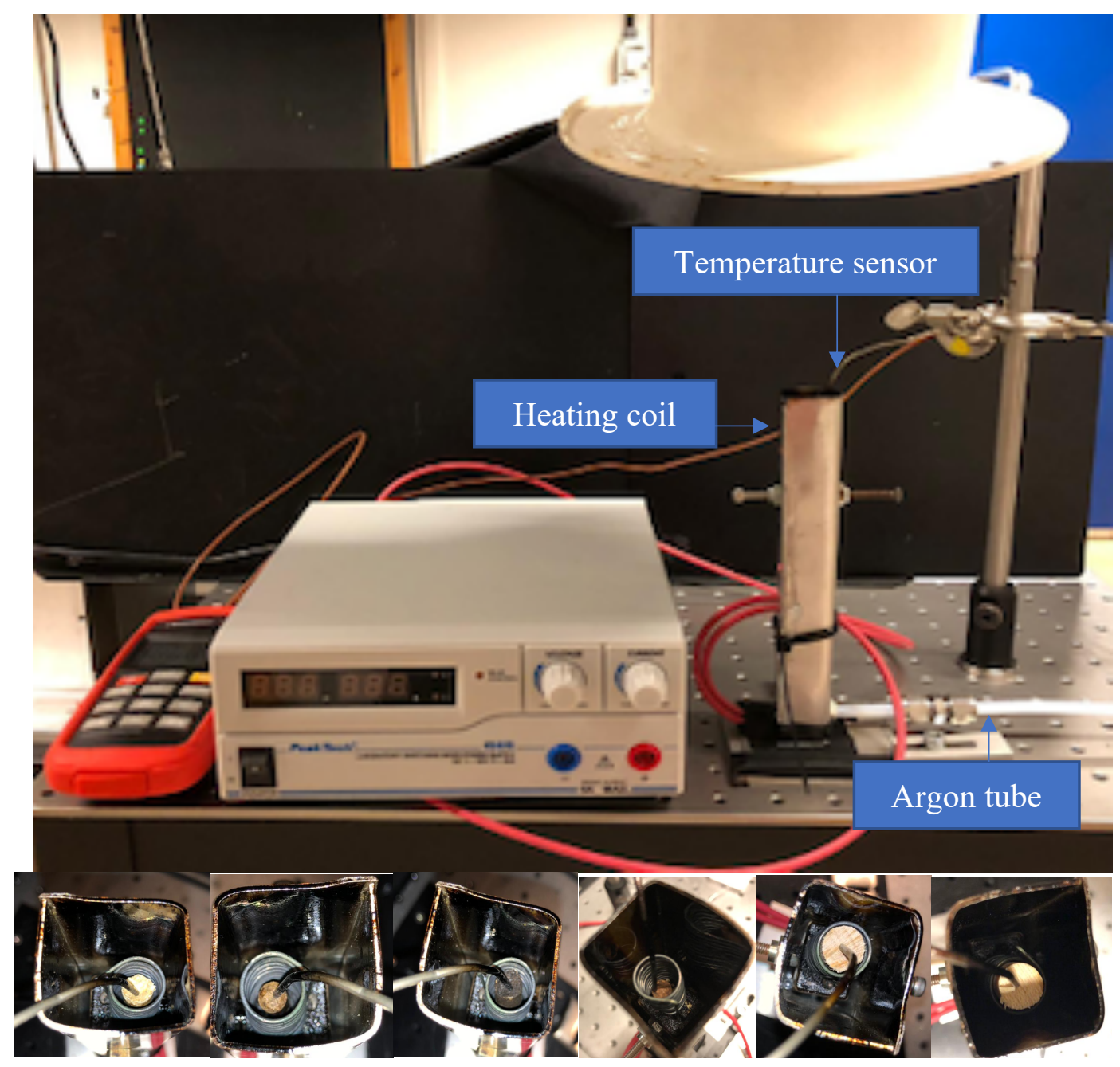

Figure 3.2 Set up of pyrolysis tools.

The heating-coil was set at fixed current and voltage $(8 \mathrm{~A}, 5.5 \mathrm{~V})$, and the temperature change was recorded during the process. Thus, the heating rate can be obtained by calculating the changing temperature over time. The position of the thermocouple has a significant impact on obtaining an accurate value. The lower the thermocouple location, the higher temperature will be acquired since it will be closer to the source of the heat. Due to the bigger size of wood samples, the sensor cannot be placed in the inner part of the coil, as given in the lower part of Figure 3.2. These six images illustrate the samples presented in Figure 3.1 and Table 3.1 before the heating process. Therefore, biomass in the form of the pellet can achieve higher temperature values than wood. The temperature measurement might be less precise. However, it is still relevant to indicate an increasing temperature of the samples. Furthermore, argon gas was flowed slowly during measurement to maintain non-oxidative conditions and prevent combustion. After the expected temperature was obtained, the coil was turned off, and the argon released faster for cooling down the sample. 
The samples properties after pyrolysis are described in Table 3.2, and the charred biomass images are given in Table 3.3. Based on the table, the biomass chars experience slow heating rate and mass loss during pyrolysis. The high temperature will produce chars with greater losses. The trend of these heating rates and mass losses are presented in Figure 3.4 and 3.5, respectively. The wood samples have mass loss higher than pellet samples. The reason could be due to the pretreatment procedures, including some low-temperature pyrolysis, applied to form a pellet and increase the energy density of the samples.

Table 3.2 Properties of Charred Materials

\begin{tabular}{|c|c|c|c|c|c|c|}
\hline \multirow[t]{2}{*}{ No } & \multirow[t]{2}{*}{ Identifier } & \multirow{2}{*}{$\begin{array}{l}\text { HTT } \\
\left({ }^{\circ} \mathrm{C}\right)\end{array}$} & \multirow{2}{*}{$\begin{array}{l}\text { Heating Rate } \\
\left({ }^{\circ} \mathrm{C} / \mathrm{s}\right)\end{array}$} & \multicolumn{3}{|c|}{ Mass (g) } \\
\hline & & & & Before & After & Loss \\
\hline \multirow{3}{*}{1.} & \multirow{3}{*}{ WP } & 350 & 2.6 & 0.463 & 0.416 & 0.047 \\
\hline & & 450 & 2.1 & 0.485 & 0.249 & 0.236 \\
\hline & & 500 & 1.6 & 0.432 & 0.090 & 0.342 \\
\hline \multirow{3}{*}{2.} & \multirow{3}{*}{139} & 350 & 2.2 & 1.117 & 0.927 & 0.190 \\
\hline & & 450 & 1.8 & 1.115 & 0.389 & 0.726 \\
\hline & & 500 & 1.2 & 1.160 & 0.287 & 0.873 \\
\hline \multirow{3}{*}{3.} & \multirow{3}{*}{233} & 350 & 1.8 & 1.219 & 0.814 & 0.405 \\
\hline & & 450 & 2.2 & 1.063 & 0.426 & 0.637 \\
\hline & & 500 & 1.6 & 1.408 & 0.372 & 1.036 \\
\hline \multirow{3}{*}{4.} & \multirow{3}{*}{266} & 350 & 2.5 & 0.569 & 0.507 & 0.062 \\
\hline & & 450 & 2.2 & 0.647 & 0.281 & 0.366 \\
\hline & & 500 & 1.7 & 0.633 & 0.155 & 0.478 \\
\hline \multirow{2}{*}{5.} & \multirow{2}{*}{ Beech } & 200 & 1.1 & 1.383 & 0.887 & 0.497 \\
\hline & & 350 & 1.4 & 1.394 & 0.356 & 1.038 \\
\hline \multirow{2}{*}{6.} & \multirow{2}{*}{ Pine } & 200 & 0.9 & 1.360 & 0.643 & 0.717 \\
\hline & & 350 & 0.9 & 1.369 & 0.301 & 1.068 \\
\hline
\end{tabular}

Table 3.3 Images of measured sample after pyrolysis

\begin{tabular}{|c|c|c|c|c|}
\hline $\begin{array}{l}\text { HTT } \\
\left({ }^{0} \mathrm{C}\right)\end{array}$ & WP & 139 & 233 & 226 \\
\hline 350 & & & & \\
\hline 450 & & & & \\
\hline 500 & & & & 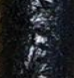 \\
\hline & & & & \\
\hline
\end{tabular}

\begin{tabular}{|c|c|c|}
\hline $\begin{array}{c}\text { HTT } \\
\left({ }^{\circ} \mathrm{C}\right)\end{array}$ & Beech & Pine \\
\hline 200 & & \\
\hline 350 & & \\
\hline
\end{tabular}




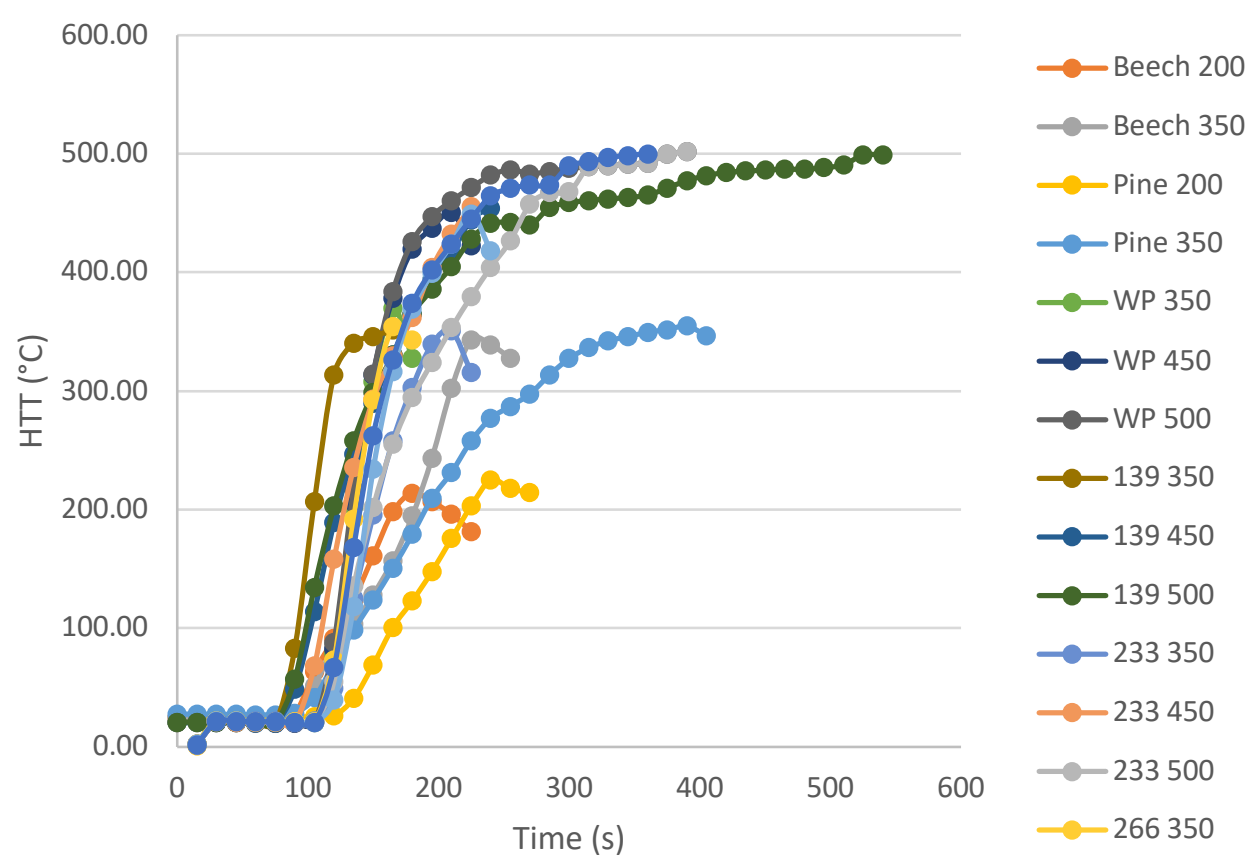

Figure 3.4. The heat rate curve for each sample.

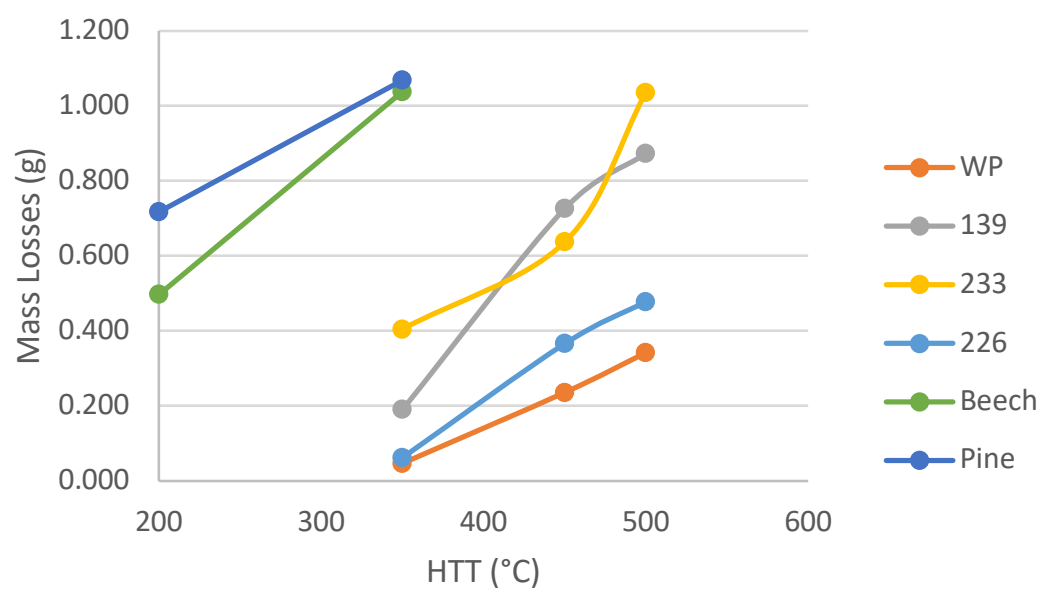

Figure 3.5 The trend of mass loss in the samples.

\subsection{Raman Spectroscopy Set Up}

Raman spectroscopy was performed by constructing a $90^{\circ}$ configuration set up, as illustrated in Figure 3.6. In this arrangement, the laser beam is directed straight to hit the sample. Then, the scattered light is detected by the spectrometer located at a perpendicular position. A waveplate is used to rotate the light polarization to be aligned with the sample structure to obtain maximum Raman signal. In order to prevent the transmitted beam to undergo random direction, it is halted by a beam dump. 


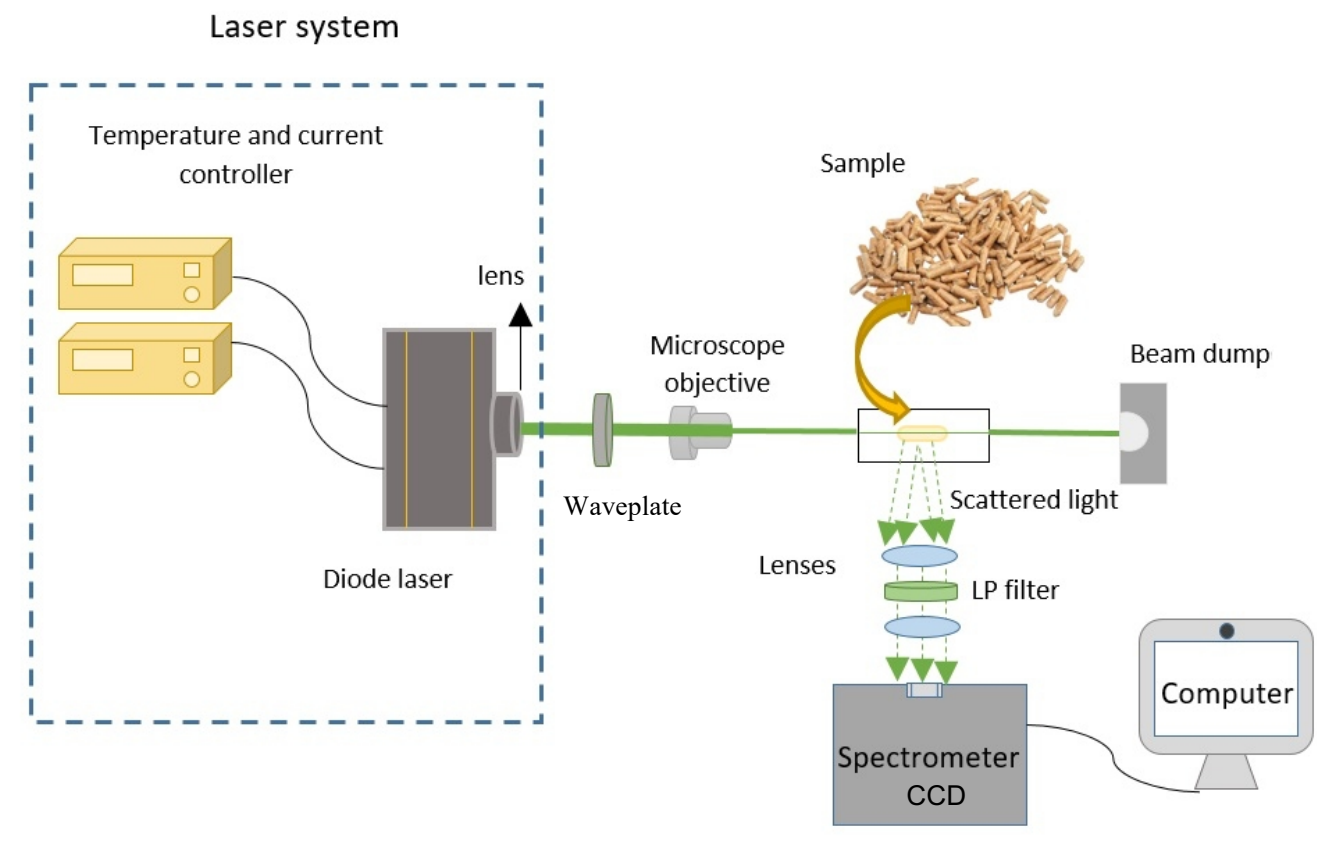

Figure 3.6. Experimental set up for Raman spectroscopy.

The laser system consists of a diode laser, which is equipped with temperature, and current controller. In the product specification, the diode laser (Thorlabs, DJ532-40) has a centre wavelength around $532 \mathrm{~nm}$, and is supplied with a maximum current of $400 \mathrm{~mA}$. The current controller is installed to adjust the output power of the laser diode. The measured power from a power meter that can be achieved by the laser for different current is presented in Table 3.3. The temperature controller has the functionality to regulate the heating of the diode laser, which is a temperature-sensitive element. A lens (Thorlabs, C171TMD-B, $\mathrm{f}=6.20 \mathrm{~mm}, \mathrm{NA}=0.30$ ) is used to focus the spread output beam into approximately $2 \mathrm{~mm}$ diameter. Furthermore, the beam is focused to micrometre scale by utilizing a microscope objective ( $\mathrm{NA}=0,10,4 \mathrm{x}$ magnification). The beam spot is greater than the carbon micro crystallites size in the chars, which is known to be approximately 2-200 $\mathrm{nm}$ [11]. Thus, a broad measurement area could be obtained and provides a fair representation of the char structure.

The interaction of beam and sample produces scattered light, which is very weak. In order to optimize the signal collection, two lenses $(\mathrm{f}=25 \mathrm{~mm}, \mathrm{f}=75 \mathrm{~mm}$ ) are used for collimation and relay of the light to the spectrometer with entrance slit around $400 \mu \mathrm{m}$. A strong Rayleigh scattering signal at wavelength $532 \mathrm{~nm}$ is separated from the Raman signal using a long-pass filter (Edge Basic BLP 01-532R-25). Finally, the resulting spectra from spectrometer (Shamrock SR-750-A-R, spectral resolution $6 \mathrm{~cm}^{-1}$ at $532 \mathrm{~nm}$ ) are registered by a CCD camera (Andor iDus DU 420A-OE, $1024 \times 255$ pixel), acquired in the computer, and available for further analysis. 
Table 3.4 Laser Power Measurement

\begin{tabular}{rccc}
\hline No & $\begin{array}{c}\text { Laser Current } \\
\text { Display (mA) }\end{array}$ & $\begin{array}{c}\text { Power Metre } \\
\text { Reading (mW) }\end{array}$ & $\begin{array}{c}\text { Standard } \\
\text { Deviation }\end{array}$ \\
\hline 1 & 0 & 1.1 & 0.058 \\
\hline 2 & 60 & 1.2 & 0.058 \\
\hline 3 & 120 & 1.2 & 0.058 \\
\hline 4 & 180 & 1.7 & 0.153 \\
\hline 5 & 240 & 3.5 & 0.100 \\
\hline 6 & 300 & 13.3 & 0.058 \\
\hline 7 & 315 & 18.6 & 0.058 \\
\hline 8 & 330 & 28.1 & 0.058 \\
\hline 9 & 345 & 33.2 & 0.231 \\
\hline 10 & 360 & 30.1 & 0.001 \\
\hline 11 & 375 & 33.4 & 0.173 \\
\hline 12 & 390 & 37.0 & 2.108 \\
\hline
\end{tabular}

In this study, a variety of measurements with different settings was performed to represent the reproducibility. The laser was adjusted for two laser powers $(18.6 \mathrm{~mW}$ and $28.1 \mathrm{~mW})$, two integration time in the CCD (30s and 60s), and two different positions in the sample structure. These set up with moderate power and integration time are selected to reduce the effect of laser heating during the measurement that can potentially cause thermal degradation to the sample. In order to get repeatability, each measurement with each setting was repeated three times. Thus, the total amount of data can be obtained for one sample in one HTT is about 24 Raman spectra. However, a separate measurement with another procedure, which involves more variation in laser power and integration time, were also conducted to identify the effect of laser power and integration time on the measurement. When the independent variable is laser power, the integration time is fixed at the 30s. On the contrary, as for integration time to act as independent variable, the laser power is adjusted to be $33.4 \mathrm{~mW}$.

\subsection{Evaluation Protocols}

The evaluation of the Raman peaks is tested by a program built in Python, as illustrated in Figure 3.7. In order to remove background noise, Raman measurement was carried out at two closely spaced wavelength in which the second spectrum had $2 \mathrm{~nm}$ wavelength shifted by the spectrometer. The two input of Raman spectra are filtered, subtracted, then integrated to produce a spectrum with background noise removed. This procedure is then followed by a baseline correction, which is manually adjusted at a fixed value. As the background typically varies for every sample, while the spectrum analysis is affected significantly by the baseline, another evaluation by 
MATLAB program, which can correct the baseline automatically, is also applied to verify the result and process the large amount of data.

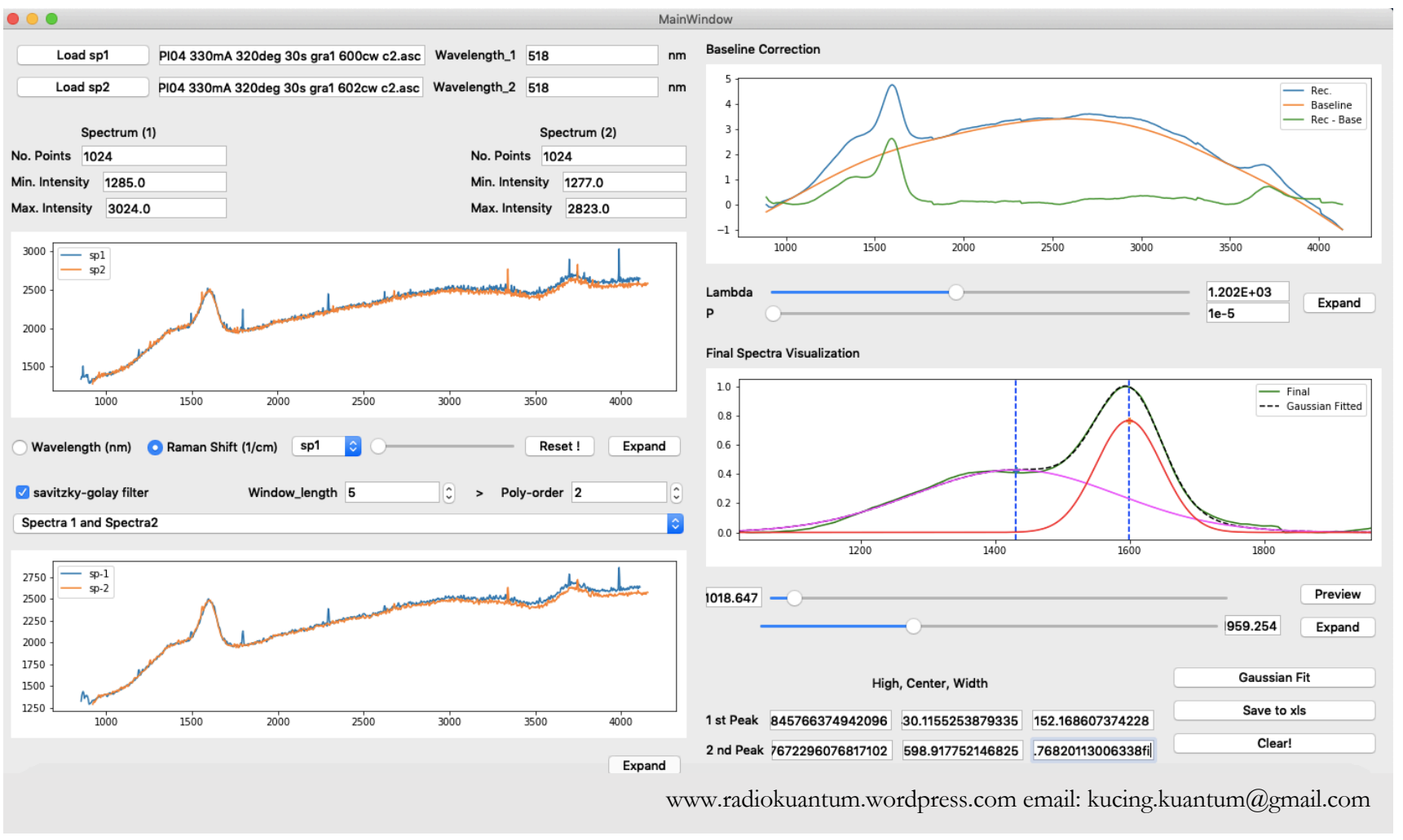

Figure 3.7 Program evaluation by Phyton main window.

A deconvolution is purposed to obtain the individual spectral peaks and their parameters from the Raman spectrum. Various protocols have been reported to analyze the first-order Raman peaks. As an example, a fitting by 1 Gaussian and 4 Lorentzian is commonly applied [14][18][30]. Other methods consisting of a greater number of peaks ( 9 peaks) were also proposed to have better identification of spectral compound in the intensities [1,31]. Likewise, a deconvolution with fewer peaks ( 2 peaks) was also found in several references $[1,18]$. In order to simplify the analysis, two Gaussian fittings were used in this study as shown in the lower right graph of Figure 3.7. Furthermore, the R-square parameter was determined to evaluate the Goodness of Fit (GoF). The value close to 1 indicates the Gaussian curves are well fitted with the spectrum. 


\section{Chapter 4}

\section{Results and Discussion}

Experimental results of all biomass samples will be presented in this chapter. Firstly, an elemental analysis of the charred materials is described. Secondly, a Raman spectroscopy evaluation follows this. Then, the analysis from both methods is compared and discussed. Furthermore, several properties such as laser power and exposure time, which affect the measurement results will be discussed in this section.

\subsection{Elemental Analysis}

Table 4.1 shows the chemical composition of biomass samples through elemental analysis. Unlike other measured samples, which were heated to varying temperatures, for the Beech and Pinewood cases, the measurement was conducted for two conditions. The first one was without pyrolysis and uncharred biomass (symbolized by temperature $0^{\circ}$ ), the other was pyrolyzed with heat temperature treatment $\left(\mathrm{HT}^{\prime} \mathrm{T}\right)$ to around $350^{\circ} \mathrm{C}$.

In order to have the same scale unit, the indexes were calculated based on Equation (2.10) and (2.11) to get values between 0 and 1 . Thus, allowing the data to be normalized in the same manner. By this standardization and discussion in reference [22], 0 has the lowest aromaticity, and the highest level is given by 1 . Before measurement, each sample from different pyrolysis temperature needs to be grinded and put in the plate consisting of some small containers. The value in the table represents the average of measurement on material from the same sample, which is placed into three different sample containers.

Tabel 4.1. Chemical composition of biomass samples

\begin{tabular}{ccccccccccc}
\hline Sample & HTT & \multicolumn{2}{c}{ Elemental Analysis amt $(\%)$} & \multicolumn{2}{c}{ Atomic Ratio } & \multicolumn{2}{c}{ Indexes $[1-0]$} & \multicolumn{2}{c}{ Indexes [0-1] } \\
\cline { 3 - 11 } Id. & $\left({ }^{\circ} \mathrm{C}\right)$ & $\mathrm{H}$ & $\mathrm{O}$ & $\mathrm{C}$ & $\mathrm{O} / \mathrm{C}$ & $\mathrm{H} / \mathrm{C}$ & $\mathrm{O}-\mathrm{C}$ & $\mathrm{H}-\mathrm{C}$ & $\mathrm{O}-\mathrm{C}$ & $\mathrm{H}-\mathrm{C}$ \\
\hline \multirow{2}{*}{ WP } & 350 & 4.73 & 25.33 & 69.94 & 0.27 & 0.81 & 0.89 & 0.91 & 0.11 & 0.09 \\
& 450 & 3.95 & 18.54 & 77.51 & 0.18 & 0.61 & 0.53 & 0.58 & 0.47 & 0.42 \\
& 500 & 2.84 & 11.99 & 85.18 & 0.11 & 0.40 & 0.24 & 0.23 & 0.76 & 0.77 \\
\hline \multirow{2}{*}{226} & 350 & 4.21 & 23.03 & 72.77 & 0.24 & 0.69 & 0.75 & 0.71 & 0.25 & 0.29 \\
& 450 & 3.75 & 17.40 & 78.85 & 0.17 & 0.57 & 0.47 & 0.51 & 0.53 & 0.49 \\
& 500 & 2.91 & 13.51 & 83.58 & 0.12 & 0.42 & 0.30 & 0.26 & 0.70 & 0.74 \\
\hline \multirow{2}{*}{233} & 500 & 4.53 & 26.01 & 69.46 & 0.28 & 0.78 & 0.92 & 0.86 & 0.08 & 0.14 \\
\hline \multirow{2}{*}{139} & 500 & 2.80 & 10.84 & 86.36 & 0.09 & 0.39 & 0.19 & 0.21 & 0.81 & 0.79 \\
\hline \multirow{2}{*}{ Beech } & 0 & 4.91 & 27.24 & 67.85 & 0.30 & 0.87 & 1.00 & 1.00 & 0.00 & 0.00 \\
& 350 & 2.02 & 5.44 & 92.54 & 0.04 & 0.26 & 0.00 & 0.00 & 1.00 & 1.00 \\
\hline \multirow{2}{*}{ Pine } & 0 & 4.73 & 25.33 & 69.94 & 0.27 & 0.81 & 0.89 & 0.91 & 0.11 & 0.09 \\
& 350 & 3.95 & 18.54 & 77.51 & 0.18 & 0.61 & 0.53 & 0.58 & 0.47 & 0.42 \\
\hline
\end{tabular}


In the theory part, it is mentioned that pyrolysis of biomass emits other gaseous products $\left(\mathrm{CO}_{2}\right.$, $\mathrm{CO}$, alkanes, alkenes, tar, and benzene), liquid products (water, tar), and solid product (char). By changing temperature, it is possible to modify the composition of the formed product [5][25]. Based on Table 4.1, the materials, which possess high carbon content can be clearly seen in the higher HT'T. On the contrary, the composition of $\mathrm{H}$ and $\mathrm{O}$ is getting lower with the rise of HTT. The reason is due to the removal of atoms $\mathrm{H}$ and $\mathrm{O}$ from the particle during the pyrolysis. As for instance, the oxygen losses are in the form of $\mathrm{CO}_{2}$ and $\mathrm{CO}$ gases. The higher temperature breaks the weaker bond within the particle structure, while the hydrogen atom is known to be reactive with many elements and compound. The heating process will give the biomass particle a tension of pressure and releases a massive volatile matter [11].

Numerous methods already established to measure the aromaticity in biomass since it is much easier to determine than the evaluation of the degree of aromatic condensation. The elemental analysis can give an indirect assessment of aromaticity through $\mathrm{M}$ indexes. This is because $\mathrm{O}-\mathrm{C}$ and $\mathrm{O}-\mathrm{H}$ indexes yield the proportion of $\mathrm{C}$ in the sample within the changing of HTT. A sequence of processes such as dehydration, depolymerization, and volatilization occur to form aromatic $\mathrm{C}$ structure, while the $\mathrm{H}$ and $\mathrm{O}$ atoms are depleted as the temperature is rising [22]. In order to portray characteristic trends of samples aromaticity, the $\mathrm{O}-\mathrm{C}$ and $\mathrm{H}-\mathrm{C}$ indexes $\left(\mathrm{M}_{\text {index [0-1] }}\right)$ are plotted versus HTT in Figure 4.1 (a) and (b).

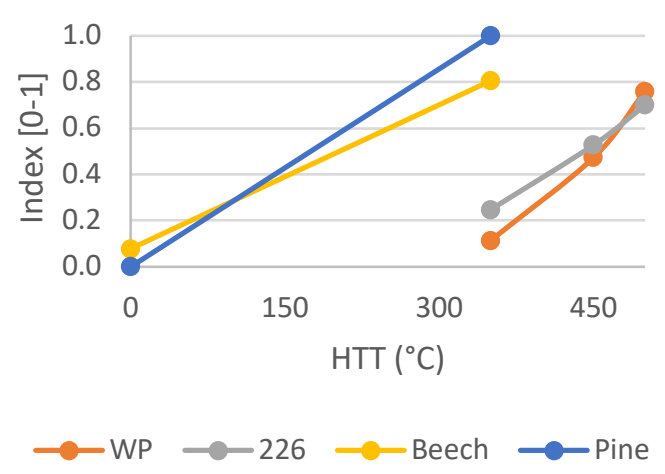

(a)

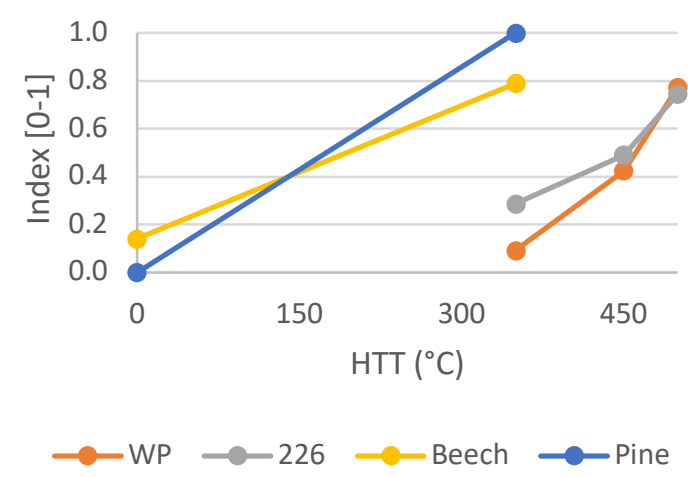

(b)

Figure 4.1 Aromaticity trend evaluated through (a) $\mathrm{O}-\mathrm{C}_{\text {index }}$ (b) $\mathrm{H}-\mathrm{C}_{\text {index }}$ from elemental analysis.

According to the graphs in Figure 4.1, either O-C index or H-C index shows the same trend. The indexes are elevating as the HTT goes up. Thus, this indicates an extensive aromatic ring formation for higher charring temperature. The rising O-C index depicts oxygen losses within the increase of HTT, or fewer O-functional groups remaining in the char. In the same way, the high value of the H-C index exhibits the large deposal of hydrogen due to the high reactivity behavior. Thoroughly, Pine char also has higher O-C and H-C index than beech in the high HTT. Pine is a softwood type, 
which contains less ash and higher amount of lignin than hardwood type such as Beech. The ash content tends to obstruct the aromatization. Meanwhile, lignin is polymers of phenylpropane units, which linked by $\mathrm{C}-\mathrm{C}$ or $\mathrm{C}-\mathrm{O}-\mathrm{C}$ bonds. The Pine char, which contains less ash and is rich content lignin, is thus more prominent to have higher aromaticity $[5,22]$.

\subsection{Raman Spectrum}

A measured spectrum of biomass char from pyrolysis is presented in Figure 4.2. A recorded signal is plotted as normalized intensity against wavenumber. In the range of the spectrum, it is noticeable from carbon origin. The first-order Raman spectrum appears as two broadened overlapped peaks in the region between $1100 \mathrm{~cm}^{-1}$ and $1800 \mathrm{~cm}^{-1}$. Moreover, the second order is weakly observed in the range of $2500 \mathrm{~cm}^{-1}$ to $3500 \mathrm{~cm}^{-1}$. Generally, the two maxima peaks are denoted as the $\mathrm{D}$ band $\left(\sim 1380 \mathrm{~cm}^{-1}\right)$ and the $\mathrm{G}$ band $\left(\sim 1580 \mathrm{~cm}^{-1}\right)$.

In an ordered carbon structure, the terms of D and G represent defect and graphite structure, respectively. As mentioned in the theory part, the $\mathrm{D}$ band has a correspondence to breathing mode of $\mathrm{sp}^{2}$ in rings, while $\mathrm{G}$ band is associated with $\mathrm{sp}^{2}$ bonded carbon stretching vibration both in rings and chains. The presence of $\mathrm{sp}^{2}-\mathrm{sp}^{3}$ bonds determines the (amorphous) carbon structure in the material. The contribution of amorphous carbon structure gives rise to overlap along 1400 $\mathrm{cm}^{-1}$ to $1500 \mathrm{~cm}^{-1}$ range, which is known as valley (V) region. As the size of the amorphous structure increases, the valley region will be more flattened.

As for biomass char, which is a more disordered structure, the notation of D and G does not relate to its graphitic structure, but more likely to the aromatic ring systems. The D band is attributed to the condensed structure of large aromatic ring systems (more than six fused rings). Meanwhile, the amorphous structure with small aromatic ring systems ( 3 to 5 fused rings) is indicated by the $G$ band [11]. Either for graphite or biomass char, the valley region with the addition of Total Raman Area (TRA) indicate the proportion of amorphous structure.

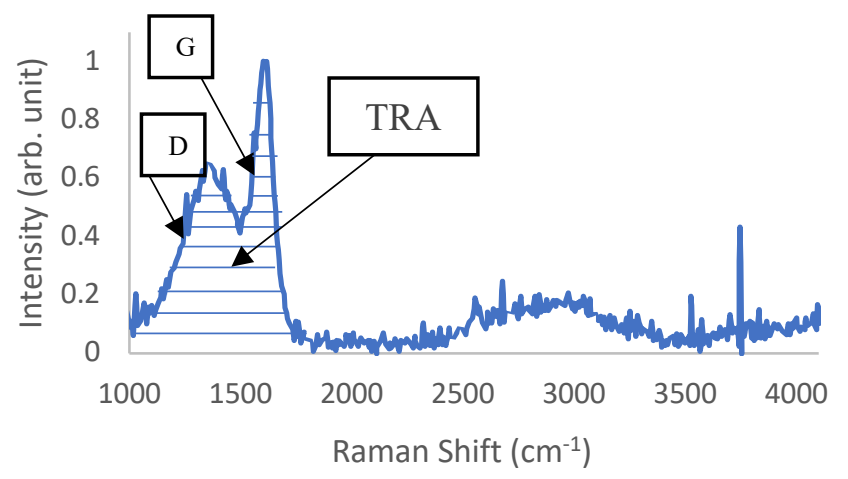

Figure 4.2. Raman spectrum of biomass char. 


\subsection{Structural Evolution}

Samples of biomass that were pyrolyzed at different temperature have structural property changes. The Raman spectra of chars from biomass pellets are given in Figure 4.3 (a), (b), (c), and (d). Meanwhile, Figure 4.4 (a) and (b) show the measured spectra of Beech and Pine chars. The intensity values are normalized by dividing with the maximum value and presented in an arbitrary unit (a.u). In brief, the measurement result shows a similar shape with D and G bands, as discussed in the previous section.

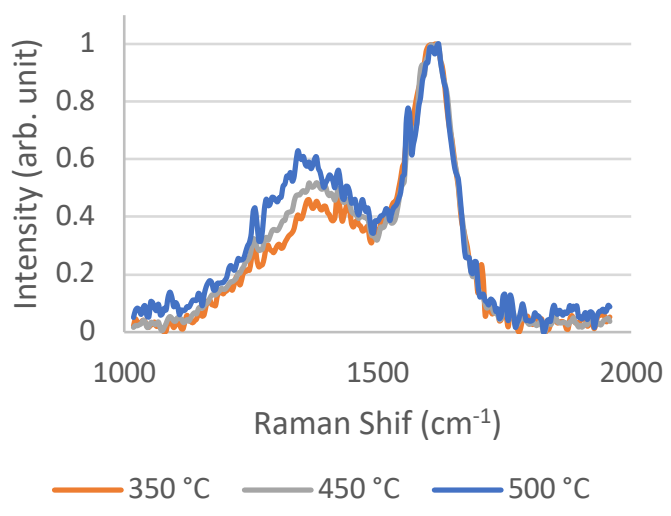

(a)

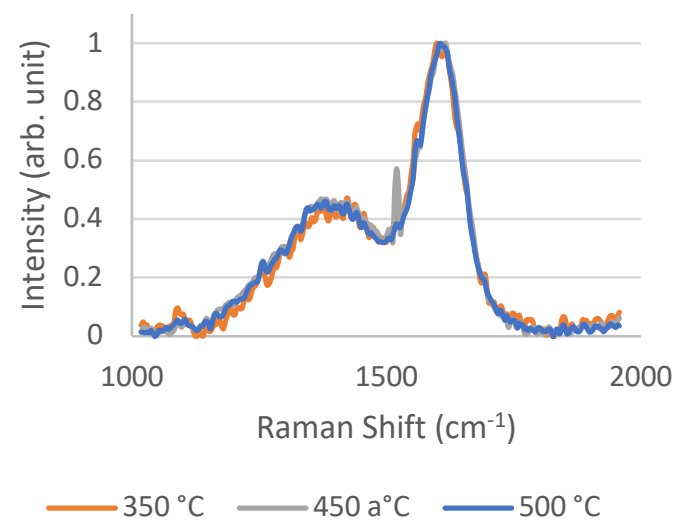

(c)

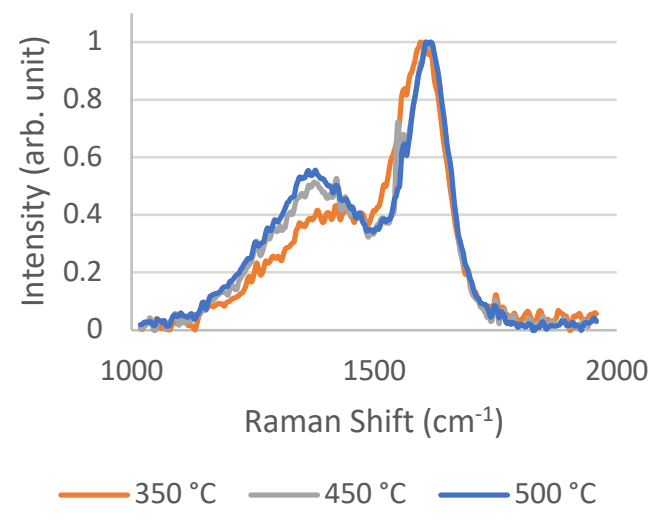

(b)

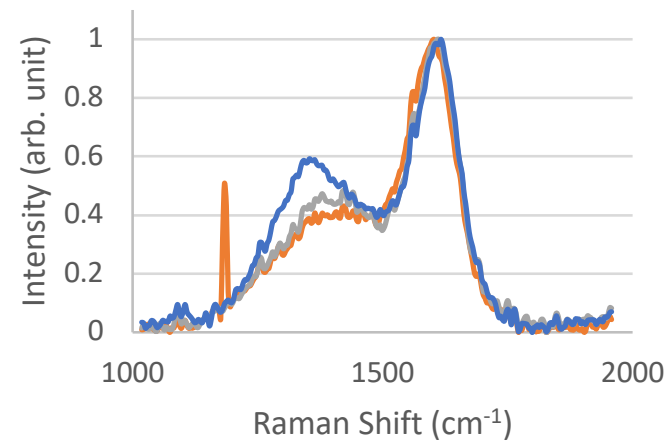

$-350^{\circ} \mathrm{C}-450{ }^{\circ} \mathrm{C}-500^{\circ} \mathrm{C}$

(d)

Figure 4.3. Structural changes in Raman spectra due to different pyrolysis temperature of biomass pellets with identification: (a) WP (b) 139 (c) 226 (d) 233. 


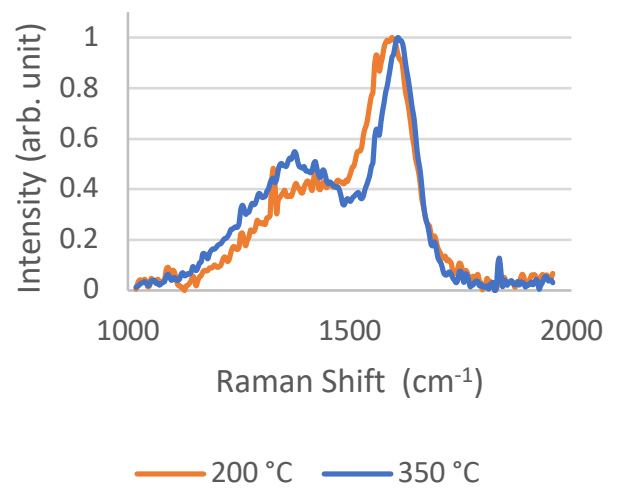

(a)

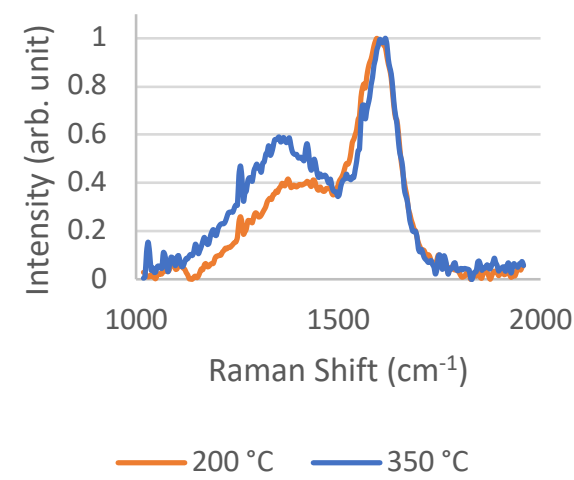

(b)

Figure 4.4. Raman spectra of pyrolyzed wood: (a) Beech (b) Pine.

As can be seen in Figure 4.3 and 4.4, Raman spectra from all the samples give spectral information about the impact of different pyrolysis treatment through the feature of the peak's height. The D band appears to be elevated as the temperature increases although the $G$ band stays the same. Thus, the height difference between the two peaks will be reduced. According to this observation, heat treatment gives change to the aromatic structure of the chars. Increasing the pyrolysis temperature would raise the aromaticity with an increased number of a larger ring structures. This alternation is mainly due to loss of the highly reactive structure such as cycloheptane and cyclooctane centered ring systems. The cyclic clusters and aromatic rings would form pyrenes [32].

Moreover, the higher temperature apparently has resulted in a more pronounced valley, while the lowered temperature possesses a flattened valley. In some spectra, for instance, at sample 139 and 233, the higher temperature also gives a sharper $\mathrm{D}$ band peak. As mentioned above, the amorphous structure is related to the valley region. It means that the contribution of the amorphous structure is higher for the lower temperature.

Quantitative measurements to analyze the effect of pyrolyzed chars were carried out by evaluating the relative height of $\mathrm{D}$ and $\mathrm{G}$ peaks from the Raman spectra. In order to get the intensity ratio (ID/IG), the Raman spectra of the chars have background removed, and the Gaussian fit applied. Figure 4.5 shows the illustration of the background subtraction and Gaussian fitting of the spectra. The plane size $\left(\mathrm{L}_{\mathrm{a}}\right)$ of the polyaromatic unit can be estimated from the ratio based on equation (2.9). The calculated result from all samples is presented in Table 4.2 and 4.3. As mentioned in the experimental chapter, the measurements were carried out from two different positions set up with different laser setting $(18.6 \mathrm{~mW}$ and $28.1 \mathrm{~mA})$ and detector setting (integration time $30 \mathrm{~s}$ and $60 \mathrm{~s}$ ). 
The values stated in the table represent the average of all measurements with three times of repetition.

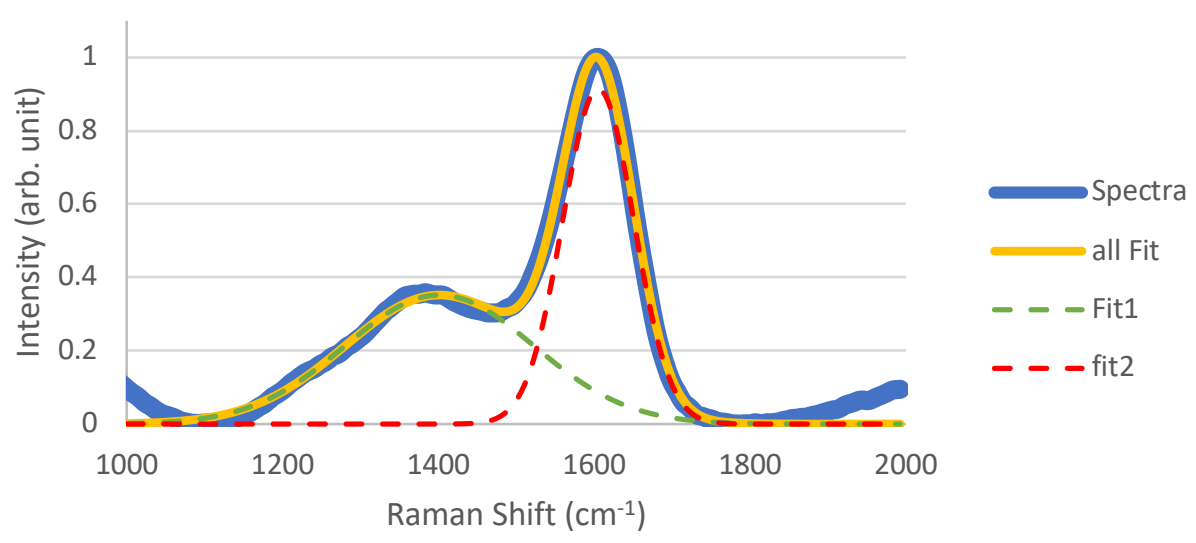

Figure 4.5. Gaussian fitting of Raman spectra with background subtraction.

Table 4.2. Structural properties of pellet chars

\begin{tabular}{|c|c|c|c|c|c|c|c|c|c|}
\hline \multirow{2}{*}{\multicolumn{2}{|c|}{$\begin{array}{l}\text { Parameter } \\
\text { Sample Id. }\end{array}$}} & \multicolumn{4}{|c|}{ Intensity Ratio (ID/IG) } & \multicolumn{4}{|c|}{$\mathrm{L}_{\mathrm{a}}(\AA)$} \\
\hline & & WP & 139 & 226 & 233 & WP & 139 & 226 & 233 \\
\hline \multirow{3}{*}{ 点 } & $350^{\circ} \mathrm{C}$ & 0.50 & 0.49 & 0.47 & 0.55 & 8.87 & 8.85 & 8.65 & 9.33 \\
\hline & $450^{\circ} \mathrm{C}$ & 0.68 & 0.58 & 0.58 & 0.60 & 10.39 & 9.57 & 9.57 & 9.72 \\
\hline & $500{ }^{\circ} \mathrm{C}$ & 0.76 & 0.65 & 0.56 & 0.73 & 11.01 & 10.17 & 9.44 & 10.79 \\
\hline
\end{tabular}

Table 4.3. Structural properties of wood chars

\begin{tabular}{crcccc}
\hline & Parameter & \multicolumn{2}{c}{ Intensity Ratio (ID/IG) } & \multicolumn{2}{c}{$\mathrm{L}_{\mathrm{a}}(\AA)$} \\
\hline \multicolumn{2}{c}{ Sample Id. } & Beech & Pine & Beech & Pine \\
\hline & $200{ }^{\circ} \mathrm{C}$ & 0.54 & 0.51 & 9.27 & 8.97 \\
\cline { 3 - 6 } & $350{ }^{\circ} \mathrm{C}$ & 0.65 & 0.77 & 10.15 & 11.06 \\
\hline
\end{tabular}

According to Table 4.2 and 4.3, the intensity ratios from all char samples exhibit increasing value as HTT is rising. Referring to the band assignation, the proportion of condensed large aromatic rings systems is increasing. The amorphous small aromatic ring systems are turned into larger ones and become condensed due to HTT. This result shows that the aromaticity in the structure of biomass chars is elevated by heat treatment. Thus, the quantitative evaluation also yields the same result as qualitative analysis by height profile inspection. A visualization of the relative intensity trend from biomass chars is illustrated in Figure 4.6.

The lateral size or plane size $\left(\mathrm{L}_{a}\right)$ is also in line with the ratio pattern. $\mathrm{L}_{\mathrm{a}}$ for chars, which has higher HTT, give larger values. This result is linked to the increased crystalline surface area as well as the porosity [18]. Those two parameters are linearly correlated and mainly influenced by pyrolysis temperature. The chars were transformed to be more structurally ordered as they were produced 
in high HTT. Thus, the rising size of aromatic planes and declining the interplanar distance would produce a larger surface area per volume [2]. Moreover, the surface properties are managed by a functional group, which is found on the aromatic sheet and integrated with aromatic rings as a heteroatom [33]. The heterogeneity is affected by biomass composition and formation condition [2]. Evolution of the structure can be conducted by providing different HTT. Furthermore, the graphite-like crystalline structure is regarded as highly disordered when the layer usually consists of 3 to 4 turbostratically stacked layers with lateral size $\left(\mathrm{L}_{\mathrm{a}}\right)$ up to $30 \AA$ with interlayer distance $\left(\mathrm{L}_{\mathrm{c}}\right)$ $3.5 \AA$ [31]. The $L_{a}$ values provided in Table 4.2 and 4.3 are around 9-10 $\AA$, and within the value $30 \AA$. They suggest that the biochars exhibit disordered structure with turbostratic arrangement, corresponding to the previous statement. The value of $\mathrm{L}_{\mathrm{a}}$ in this study is obtained indirectly by calculation from parameter ID/IG. To be more precise, it can be compared with results from other methods such as High-Resolution Transmission Electron Microscopy (HRTEM) and X-Ray Diffraction (XRD).

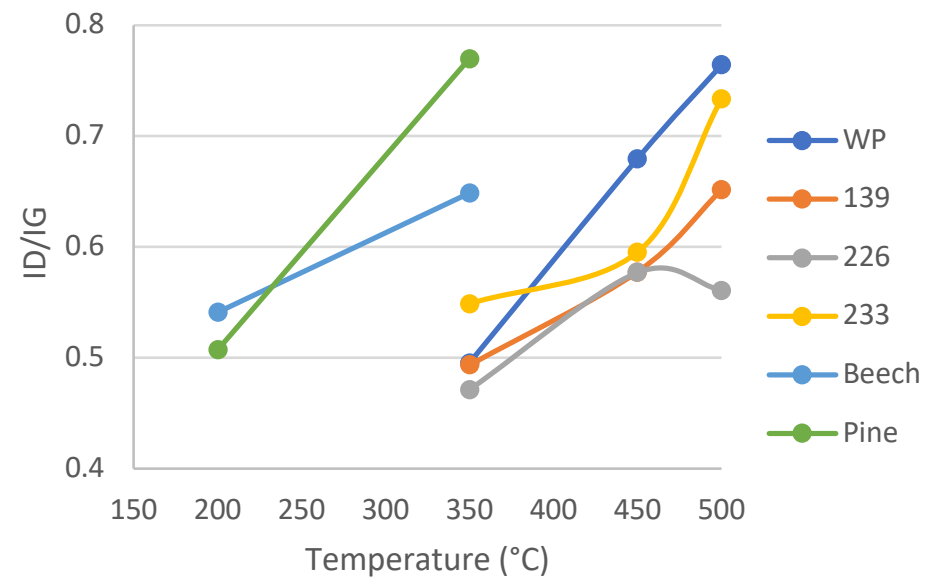

Figure 4.6. The pattern of the spectral intensity ratio of chars due to temperature difference upon pyrolysis.

Figure 4.6 express a similar pattern for all charred materials as expected. Overall, the ID/IG ratio has an increasing trend as the temperature is rising. Only the sample id 226 has slightly declined in the temperature of $450^{\circ} \mathrm{C}$ and $500^{\circ} \mathrm{C}$. It could be understandable since the final temperature has a small difference, and also the temperature measurement could be less precise. Observing closely for Pine and Beech case, Pine has a higher ratio value in the upper HT'T, which means that Pine acquires more of aromaticity. The reason is the chemical composition that has been explained in the elemental analysis part. In addition, the different cell structure of hardwood (Beech) and softwood (Pine) might give an impact in the aromaticity. Softwood type has a tracheid structure in the cell wall [34]. Thus, during the pyrolysis process, the hardwood exposes less volatile matter compared to softwood and produce lower aromaticity in the charred material. The same result also can be seen for the WP char that has higher aromaticity than the 226 char in the upper HTT. 
Comparing elemental analysis and Raman spectroscopy evaluation, both methods give results, which are in a good agreement. In terms of aromaticity, elemental analysis and Raman spectroscopy provide the measurement of biomass sample that has an increasing trend of aromaticity as the HTT rising. Indeed, the softwood is proven to have higher aromaticity than hardwood type in the upper temperature. Both methods have a different source of origin and procedure for assessment. The way of elemental analysis expresses the aromaticity is through the proportion of $\mathrm{C}$ content in the particle and require sample preparation e.g. grinding. As for the Raman technique, the sample can be directly measured. The relative intensity of two overlapped Raman peaks gives a value, which depicts the proportion of aromatic ring systems in the structure. Moreover, the plane size $\left(\mathrm{L}_{\mathrm{a}}\right)$ can be obtained from the ratio values and represent the crystalline surface area of the chars. In short, evaluation of Raman spectra can give the whole description of the degree of carbonization in the biomass chars since both parameters aromaticity and crystalline surface area are acquired [21].

A combination of chemical composition from elemental analysis and Raman structural parameter is presented in Figure 4.7. The possible correlation between the two methods is investigated further. In this figure, the ratio of valley and D band intensity is linked with logarithmic of the atomic ratio. The evaluation is performed from the sample id. WP to give an overview since any literature regarding this evaluation has not been developed yet. The data are found to be linearly proportional with the goodness of fit close to 1 . This result indicates that the chars were adjusted to turbostratic through the formation of a condensed aromatic structure. The departure of atoms $\mathrm{H}$ and $\mathrm{O}$ are correlated to this evolution [32]. A more detailed assessment is required to confirm the result with other samples. Thus, the analysis will give a better understanding of the characteristic of biochar.

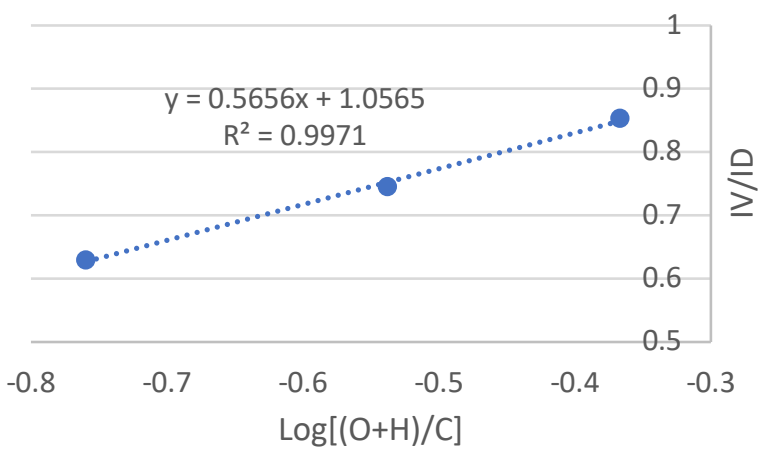

Figure 4.7 Ratio IV/ID as function of the $\log \left[\frac{\mathrm{O}+\mathrm{H}}{\mathrm{C}}\right]$ from sample id WP. 


\subsection{Comparison to Soot Spectrum}

Measurement of soot from a basic candle flame is presented in Figure 4.8 and the structural properties of soot are given in Table 4.4 .

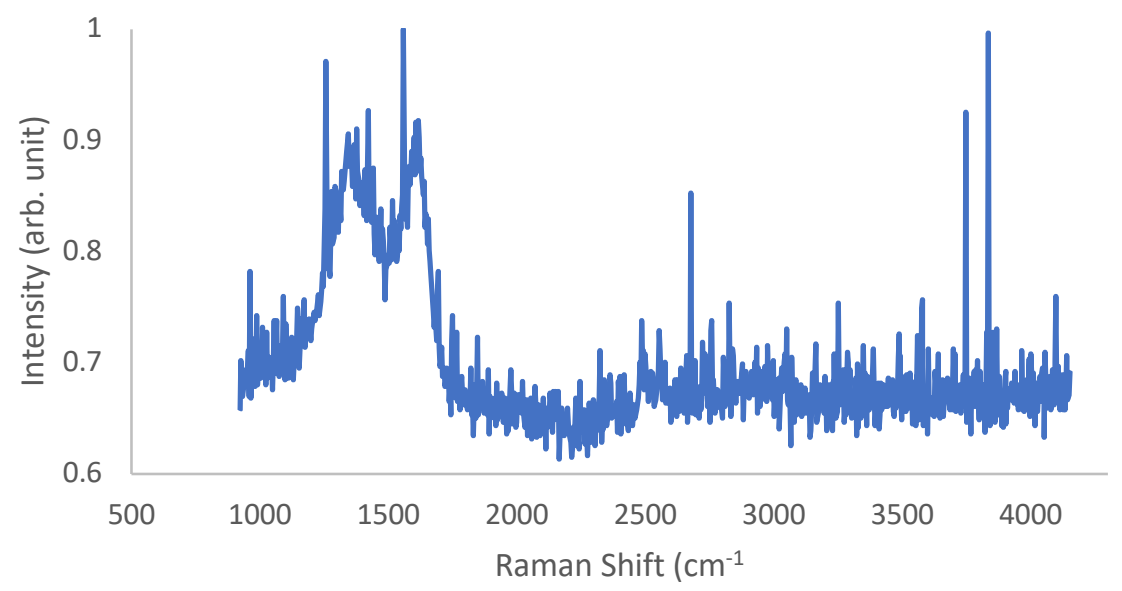

Figure 4.8 Raman spectrum of soot.

As can be seen in Figure 4.8, the soot has more noise compared to the biochar spectrum. It is because of the very weak signal of soot. The spectrum appears in the same wavelength range as biochar as expected since it is also a carbon-based material. The generation of a second order is weakly seen in the range $2500-3500 \mathrm{~cm}^{-1}$. Visually, the soot spectrum has a distinctively different shape from biochar. The two overlapped peaks appear almost at a similar height, unlike the biochar.

Table 4.4 Properties of soot

\begin{tabular}{ccc}
\hline \multirow{2}{*}{ Sample Id. } & \multicolumn{2}{c}{ Parameters } \\
\cline { 2 - 3 } & Intensity Ratio (ID/IG) & $\mathrm{L}_{\mathrm{a}}(\AA)$ \\
\hline Soot & 1.05 & 12.30 \\
\hline
\end{tabular}

The given value represents the average from all measurement that has been performed for several settings and taken at three times. It is revealed that soot has the largest value of the relative intensity and $\mathrm{L}_{\mathrm{a}}$ compared with biochar. Thus, it indicates that soot has formed a more condensed aromatic ring system and produced larger crystalline surface area. This result expresses that the degree of carbonization in soot is higher than in biochar.

\subsection{Effect of Measurement Set-Up}

\subsubsection{Laser Power}

The effect of laser power on the measurement is presented in Figure 4.9 and 4.10 


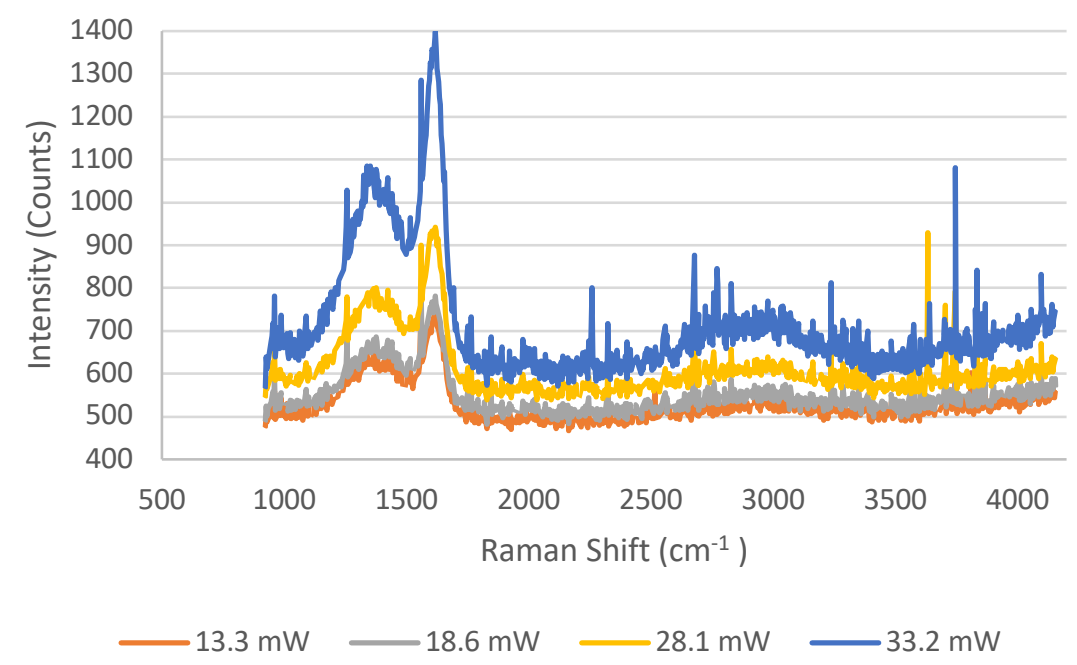

Figure 4.9 Raman Spectra of Pine char with $\mathrm{HTT}^{\circ} 350^{\circ} \mathrm{C}$ for different laser power.

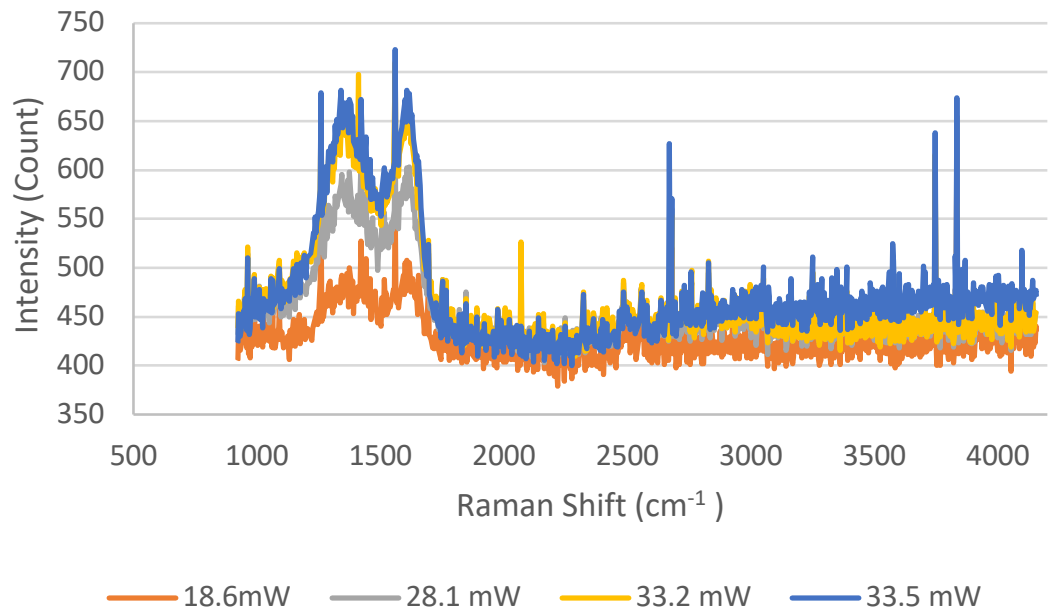

Figure 4.10 Raman Spectra of soot for different laser power.

The increasing laser power escalates the amount of measured intensity as well as the noise. This result corresponds to the theoretical knowledge of Raman signal, which is power dependent. As for the soot sample, the minimum power is not set to be $13.3 \mathrm{~mW}$ because it is not enough to generate the spectrum. In opposite, the biochar is not continued to go further than $33.5 \mathrm{~mW}$ due to fluorescence generation and Rayleigh scattering that overshadows the Raman signal. Biochar tends to have higher signal than soot, although the applied laser power is the same. The reason could be because soot sample is more carbonized and absorbs most of the laser light. It is also suggested that biochar might have larger Raman cross-section depending on the material structure and surrounding of the vibrating bonds. Roughly estimated, the signal strength for biomass is almost larger than soot signal. To obtain proper understanding, requires a more thorough measurement. 
Looking at biochar spectra, a steep raise from laser power $28.1 \mathrm{~mW}$ to $33.2 \mathrm{~mW}$ is noticeable. Continuing to increase the power can cause the char spectrum to be influenced by the laser frequency signal (Rayleigh scattering). It is observed mostly for biochar with the lowest HTT, that the shape of the Raman spectra is influenced a lot by higher laser power. As shown in Figure 4.11, the left side of the spectrum of biochar with lowest HTT appears noisy for higher laser power. Apparently, the higher laser power can heat the immature biochar, and this process can affect the generation of the spectrum.

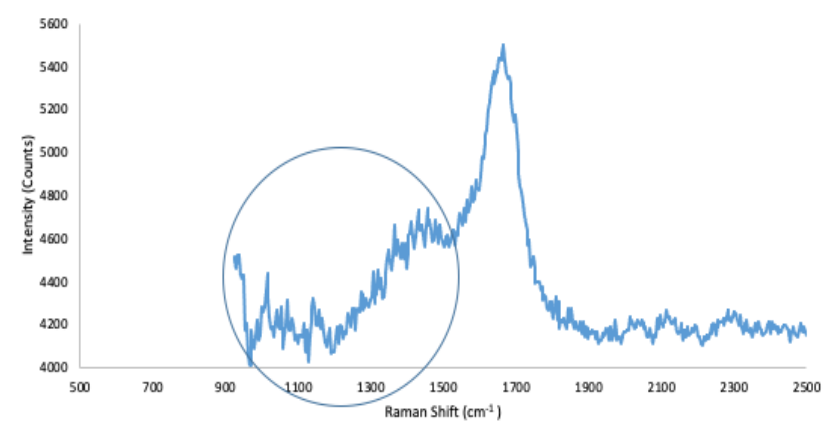

Figure 4.11. Raman spectrum of Pine char with lower HTT $\left(200^{\circ} \mathrm{C}\right)$ is affected by laser power.

Since both Raman and Rayleigh signals are power dependent, as mentioned earlier, increasing the laser power will elevate both signals. Rayleigh scattering is on the order of more than a thousand times higher than the Raman signal. Thus, the stray light from the laser signal could cover the Raman spectrum. Additionally, the frequency of laser source is around $532 \mathrm{~nm}$, which is not in the long-wavelength range. It will produce a small shift and closer distance with the laser signal, the carbon spectrum appears at around $560 \mathrm{~nm}$, which is approximately $30 \mathrm{~nm}$ apart from the laser. So, the chance that the Raman spectrum is affected by the laser signal is also high. The presence of additional unidentified peaks as the power increases could also disrupt the Raman signal as presented in Figure 4.12.

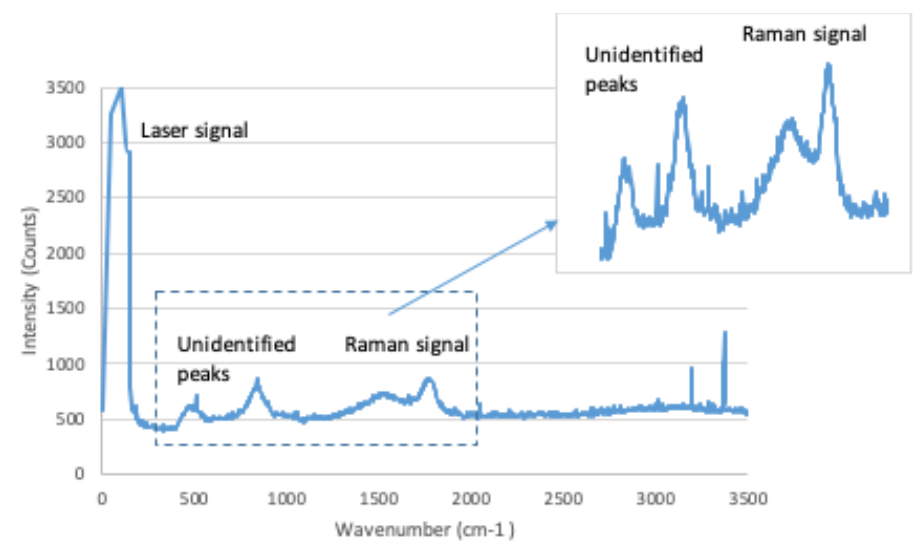

Figure 4.12. Interference from unidentified peaks to the carbon Raman spectrum in the Pine char. 
The obstruction from the laser signal should be concerned. However, for soot case, the increasing power does not seem to change the signal shape. The Raman signal is gradually increased as the laser-powered up. The reason could be due to the degree of carbonization of soot is higher than biomass. Then, the Raman signal of soot has little affection from laser heating since the carbon structure is more mature or condensed. The biochar with upper HTT also shows similar behavior, and it is less affected by the higher power since the higher HTT produce more mature carbon than the lower HTT. The distraction to the Raman signal when the power level up depends on the degree of carbonization of the material structure, the more mature samples will get less influenced.

\subsubsection{Integration Time}

Since the obtained spectra are noisy, the experiment is conducted with different CCD integration time. The measured spectra of some samples with a variation of time are presented in Figure 4.13, 4.14, and 4.15. The sample is measured with the same laser power, which is $33.5 \mathrm{~mW}$.

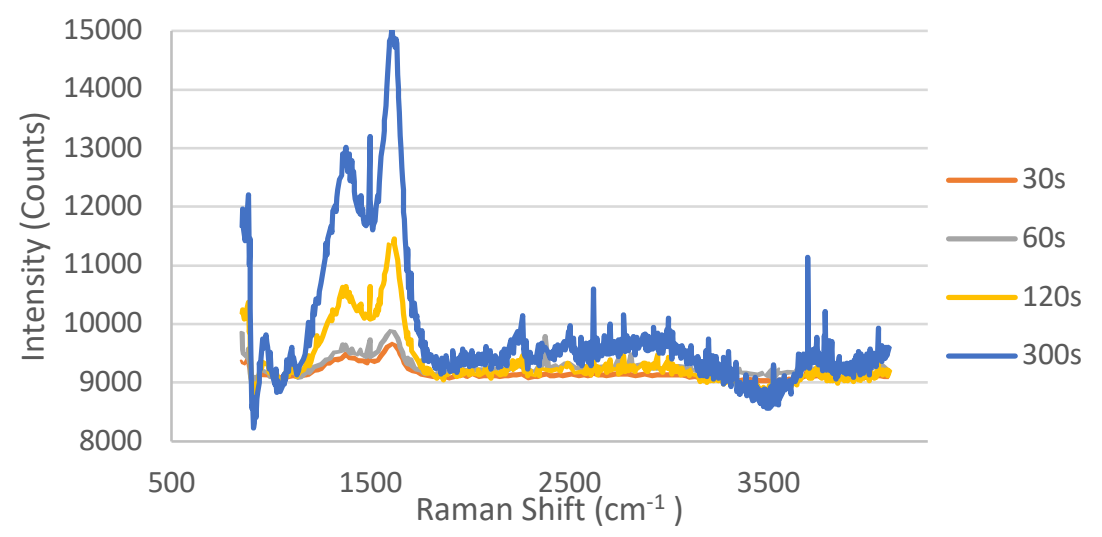

Figure 4.13. Spectra of sample id. 226 with $\mathrm{HTT}^{\top} 500^{\circ} \mathrm{C}$ in various integration time.

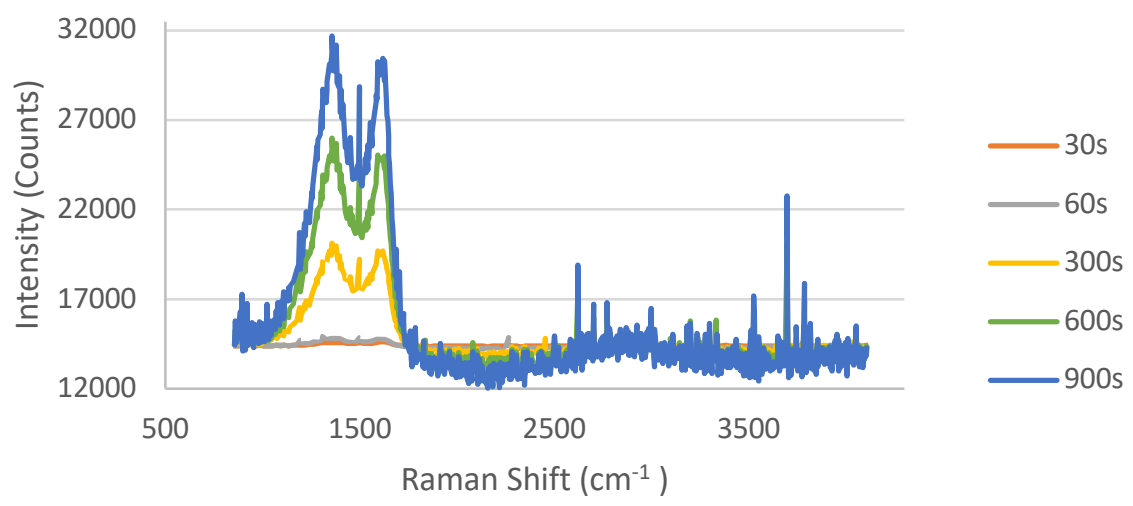

Figure 4.14. Spectra of soot sample with variation in integration time. 


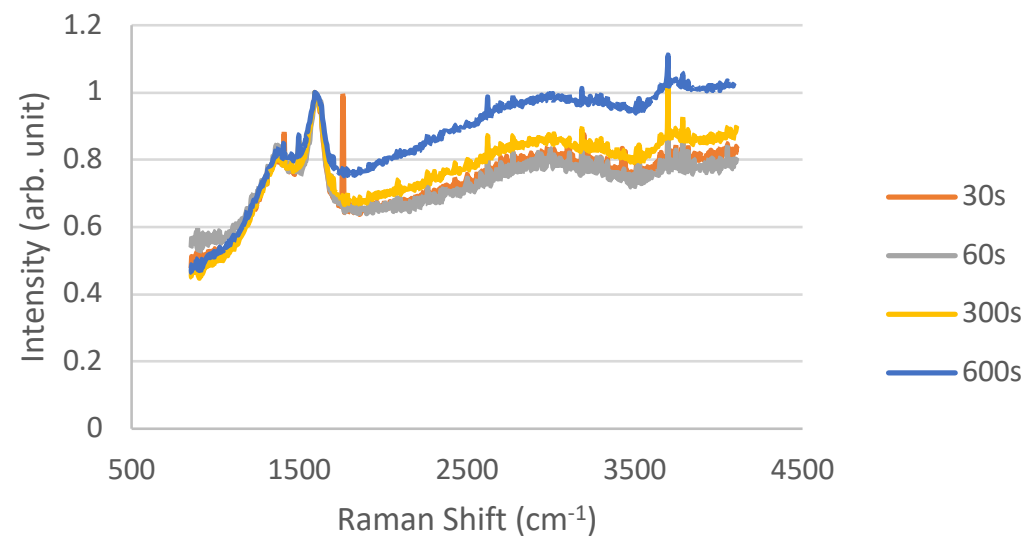

(a)

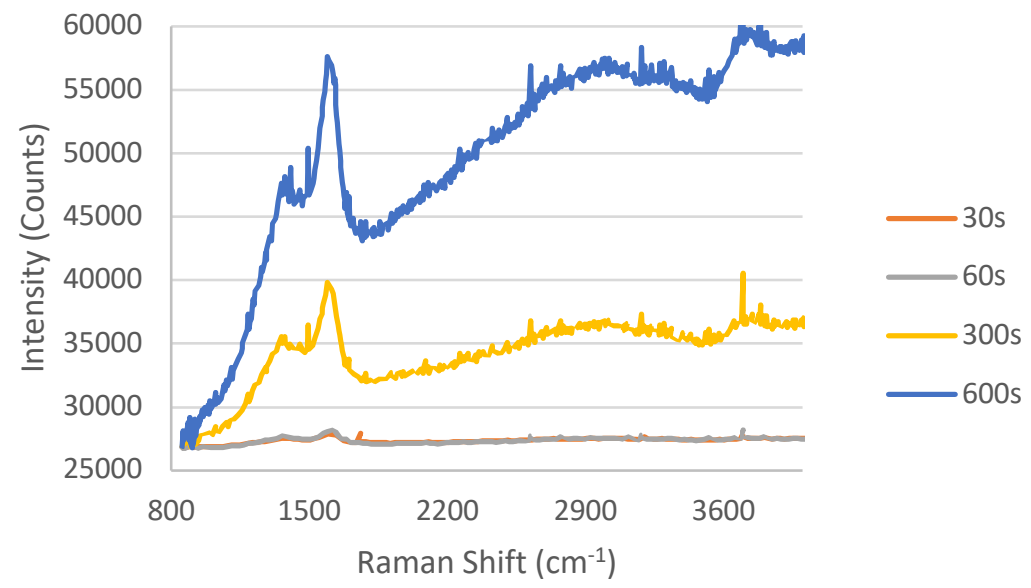

(b)

Figure 4.15. Spectra of Beech wood with HTT $350^{\circ} \mathrm{C}$ for different integration time (a) with intensity normalization (b) without intensity normalization.

Similar to higher laser power, the longer integration time will give higher intensity. The longer acquisition time, the more photons will be obtained and augment the intensity counts. Measuring with longer integration time should suppress noise. The measurement from integration time between $2 \mathrm{~s}$ to $30 \mathrm{~s}$ indicate improve signal-to-noise (SNR) ratio as presented in Figure 4.16. However, increasing from the 30 s to the higher time do not appear to reduce the noise distinctively as illustrated in Figure 4.13, 4.14, and 4.15.

Measurement of biochar spectra with longer integration time will raise the attribution of fluorescence continuously and eventually saturate the detector. That is why biochar samples cannot achieve as long integration time as soot. This evidence is shown in Figure 4.14, where the longest time gives the most inclined spectrum with stronger fluorescence background. The fluorescence background changes with integration time. Examining the entire set of the spectra, the biochars with lowest HTT tend to be highly affected. The reason is similar to the explanation 
already mentioned for the laser power. The lower HTT will produce less carbonized or immature char. Partially, the material will have reactive elements and react during the period of time. Thus, fluorescence is generated in the measurement.

Overall, the source of influenced factors cannot be treated separately. All together will have an impact on the measurement, whether it is the laser power or integration time. For example, higher laser power and longer integration time will give a spectrum with a lot of fluorescence, noise, and interference by the laser source. Thus, determine the effective time and moderate power in the measurement are necessary. Even if the post-processing can eliminate those obstacles, having an appropriate measurement set up is required to get adequate results.

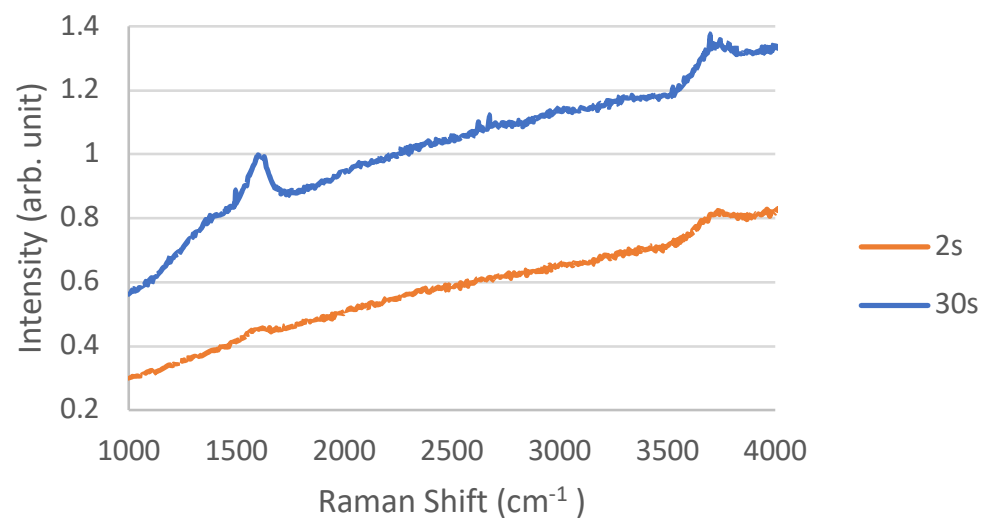

Figure 4.16. Comparison of biochar spectra at 2s and 30s CCD integration time.

\subsection{Measurement Validity}

Validity represents how well the measurement can produce reliable results. There are many factors in order to obtain a valid value in the measurement process. First, it can be from the performance of the measurement instrument itself, such as the spectrometer resolution, the ability of temperature controller to stabilize the laser, laser frequency calibration, and so on. Next, the imperfection of the instrument arrangement can give a non-optimum measurement result. For example, if the optics components are not well aligned, then the light collection is not optimized. Some parameters set up, such as suitable laser power, CCD integration time, and polarization will help to maximize the signal generation. Having measurement repeatability and reproducibility will reduce the measurement error. The standard deviation of the measurements results presented in Table 4.5. 
Table. 4.5. Standard deviation of ID/IG

\begin{tabular}{|c|c|c|c|c|c|c|c|c|c|}
\hline \multirow{2}{*}{\multicolumn{2}{|c|}{$\begin{array}{l}\text { Parameter: } \\
\text { Sample Id. : }\end{array}$}} & \multicolumn{8}{|c|}{ Standard Deviation } \\
\hline & & WP & 139 & 226 & 233 & & Beech & Pine & Soot \\
\hline \multirow{3}{*}{ 焉 } & $350{ }^{\circ} \mathrm{C}$ & 0.026 & 0.058 & 0.021 & 0.017 & $200^{\circ} \mathrm{C}$ & 0.032 & 0.052 & \multirow{3}{*}{$\begin{array}{c}0.070 \\
\text { (no HTT) }\end{array}$} \\
\hline & $450^{\circ} \mathrm{C}$ & 0.019 & 0.026 & 0.015 & 0.022 & $350^{\circ} \mathrm{C}$ & 0.025 & 0.069 & \\
\hline & $500{ }^{\circ} \mathrm{C}$ & 0.046 & 0.064 & 0.026 & 0.064 & & & & \\
\hline
\end{tabular}

Examine the result in Table 4.5. the standard deviation of the measurement pronounced small values bellow 0.1 . This value indicates that the measurement variance is not spread widely. Lastly, in the post-processing process, noise filtering, baseline correction, and a curve fitting also have an essential role for the resulted values. How close the selected Gaussian curve can represent the Raman spectrum should be quantified. In order to evaluate the goodness of fit, the determination coefficient $\left(\mathrm{R}^{2}\right)$ is obtained and given in Table 4.6. This coefficient describes the variance proportion from the dependent variable over the independent one.

Table. 4.6. $\mathrm{R}^{2}$ calculation results

\begin{tabular}{|c|c|c|c|c|c|c|}
\hline & Parameter: & \multicolumn{5}{|c|}{$\mathrm{R}^{2}$} \\
\hline & ample Id. : & WP & 139 & 226 & 233 & Soot \\
\hline \multirow{3}{*}{ 恶 } & $350^{\circ} \mathrm{C}$ & 0.993 & 0.992 & 0.987 & 0.998 & \multirow{3}{*}{$\begin{array}{c}0.991 \\
\text { (no HTT) }\end{array}$} \\
\hline & $450^{\circ} \mathrm{C}$ & 0.997 & 0.996 & 0.997 & 0.998 & \\
\hline & $500^{\circ} \mathrm{C}$ & 0.995 & 0.997 & 0.997 & 0.994 & \\
\hline
\end{tabular}

The value of $\mathrm{R}^{2}$ in Table 4.6 is getting towards to 1 , which means that the fitted curve becomes close to the original spectrum and well suited. The $\mathrm{R}^{2}$ results from the samples also do not vary significantly. The goodness of fit remains within $1 \%$ from an ideal $\mathrm{R}^{2}$. The measurement results are proven compatible with both theoretical literature and other experiments [18]. Based on these evidences, the measurement results are reliable. 


\section{Chapter 5}

\section{Conclusion and Outlook}

\subsection{Conclusion}

In this study, the elemental analysis and Raman spectroscopy give the same trend for characterizing biochar structure for pyrolysis at the different temperature. Both methods indicate that the aromaticity of biochar increase as the HTT gets higher. The alteration mainly due to release of the volatile matter in the material. Moreover, in the upper HTT, the softwood shows higher aromaticity compared to hardwood type due to the different structure of cell wall. By the Raman technique, the in-plane size $\left(\mathrm{L}_{\mathrm{a}}\right)$ of the biochar can be obtained from relative intensity of ID and IG. The $L_{a}$ value, which represents the porosity of biochar indicates larger crystalline surface area for higher HTT. Thus, Raman spectroscopy provide more detailed evaluation of the degree of carbonization than the elemental analysis in the biochar since both parameters aromaticity and crystalline surface area can be acquired. Further evaluation to link the result from the two methods are conducted by plotting the IV/ID and logarithm of the atomic ratio. A linear correlation is obtained with GoF close to 1 . This result indicates that the formation of condensed aromatic structure (crystalline phase) of the biochar has turbostratic arrangement. This evolution is related to the losses of atom $\mathrm{H}$ and $\mathrm{O}$ as the HTT increase.

The soot spectrum expressed distinct different shape from biochar spectrum with the $\mathrm{D}$ and $\mathrm{G}$ peaks appearing almost at similar height. The soot sample has larger crystalline surface area and aromaticity. Thus, soot is more carbonized than biochar since it has higher value for both parameters (the relative intensity and $\mathrm{L}_{\mathrm{a}}$ ). Moreover, the Raman spectrum of soot has more noise than biochar. The soot signal is weaker than that of biochar, probably because it absorbs more light and that the Raman cross section of biochar could be larger than soot.

The Raman measurement is influenced by setting of laser power and CCD integration time. Increasing the laser power will raise the Raman signal but also the noise. It is correlated with theoretical knowledge of Raman signal, which is power dependent. As for biochar sample with lowest HTT, the elevating power cause the fluorescence background to dominate over the signal. The longer acquisition time also increases the Raman signal since it is related to the duration of photon collection. Measurement for integration time up to 30 s indicate pronounced noise suppression, however longer than 30s do not appear to reduce the noise distinctively. For the same 
case of lowest HTT biochar, the longer integration time will raise the fluorescence contribution. This is because the lowest HTT biochar produces a less carbonized structure compared to biochar with higher temperature and soot sample. Thus, applying moderate power and integration time is preferable to obtain spectrum with noise reduction and less fluorescence impact.

\subsection{Outlook}

Characterization of biochars can be followed up by further investigation to obtain thorough assessment. More sample measurements and temperature variations to connect between measurement results from the Raman method and elemental analysis will aid to establish equation relations. Examine several points in the same biochar sample could give better understanding of the structural properties and variations within the sample. The limitation to perform temperature measurement in the pyrolysis procedure should be improved to get an accurate value of the HTT and improve comparison between different types of samples. Furthermore, managing the independent variable cautiously such as sample size will ensure to have more controllable measurement.

The usage of different excitation wavelength, which has been proposed in other study could be interesting to utilize for acquiring more precise $\mathrm{L}_{\mathrm{a}}$ calculation since it could not be done in this study. Trials with $638 \mathrm{~nm}$ laser excitation resulted in very strong background signals from Rayleigh scattering and fluorescence. Moreover, Raman measurement can be synchronized with another technique as for instance XRD or electron microscopy techniques to compare and verify the $\mathrm{L}_{\mathrm{a}}$ value. Performing measurements to obtain the Raman cross section would confirm the possibility of biochar having higher signal than soot is due to that reason. Additional parameters instead of $\mathrm{L}_{\mathrm{a}}$ should be considered to evaluate biochar structure. Lastly, the second order of Raman spectrum, which is weakly seen in this experiment is a prominent subject to explore the aromaticity of the samples in different region. 


\section{Acknowledgement}

I would like to thank my supervisors, Christian and Frederik who patiently guide and encourage me during my thesis work. Since spectroscopy is a new field for me, the learning process could be harder without their help. I also would like to thank RISPRO scholarship that give me a chance to pursue my Master Degree program in Physics, Photonics Lund University with full financial aid through the end of my studies. Thank you to my colleague Yijing Mao who cherish me during this project work, and a special thanks to my beloved family for their wishes, support, and understanding during my studies. 


\section{References}

[1] M. W. Smith et al., "Structural analysis of char by Raman spectroscopy: Improving band assignments through computational calculations from first principles," Carbon N. Y., vol. 100, pp. 678-692, 2016.

[2] A. V. McBeath, "Aromatic Condensation of Black Carbon: Its measurement and importance," Dr. Thesis Univ. Adelaide, no. March, 2012.

[3] Swedish Energy Agency, "Consequences of an increased extraction of forest biofuel in Sweden," 2014.

[4] K. Andersson, Bioenergy-the Swedish experience. How bioenergy became the largest energy source in Sweden. 2012.

[5] R. Z. Vigouroux, "PYROLYSIS OF BIOMASS • Rapid Pyrolysis at High Temperature • Slow Pyrolysis for Active Carbon Preparation,” p. 113, 2001.

[6] https://www.soderenergi.se/english/(Visited on 04/05/2020)

[7] R. K. Dash, G. Yushin, and Y. Gogotsi, "Synthesis, structure and porosity analysis of microporous and mesoporous carbon derived from zirconium carbide," Microporous Mesoporous Mater., vol. 86, no. 1-3, pp. 50-57, 2005.

[8] M. J. Bleda-Martínez, J. M. Pérez, A. Linares-Solano, E. Morallón, and D. Cazorla-Amorós, "Effect of surface chemistry on electrochemical storage of hydrogen in porous carbon materials," Carbon N. Y., vol. 46, no. 7, pp. 1053-1059, 2008.

[9] H. Zhang, W. Xiao, W. Zhou, S. Chen, and Y. Zhang, "Hierarchical porous carbon derived from sichuan pepper for high-performance symmetric supercapacitor with decent rate capability and cycling stability," Nanomaterials, vol. 9, no. 4, 2019.

[10] C. R. Correa and A. Kruse, "Biobased functional carbon materials: Production, characterization, and applications-A review," Materials (Basel)., vol. 11, no. 9, 2018.

[11] M. Asadullah, S. Zhang, and C. Z. Li, "Evaluation of structural features of chars from pyrolysis of biomass of different particle sizes," Fuel Process. Technol., vol. 91, no. 8, pp. 877$881,2010$.

[12] J. S. Lupoi, E. Gjersing, and M. F. Davis, "Evaluating lignocellulosic biomass, its derivatives, and downstream products with Raman spectroscopy," Front. Bioeng. Biotechnol., vol. 3, no. APR, pp. 1-18, 2015.

[13] X. Liu, Y. Zheng, Z. Liu, H. Ding, X. Huang, and C. Zheng, "Study on the evolution of the char structure during hydrogasification process using Raman spectroscopy," Fuel, vol. 157, pp. 97-106, 2015. 
[14] B. Manoj, "Synthesis and characterization of porous, mixed phase, wrinkled, few layer graphene like nanocarbon from charcoal," Russ. J. Phys. Chem. A, vol. 89, no. 13, pp. 24382442, 2015.

[15] R. Krishnan, J. John, and B. Manoj, "Raman spectroscopy investigation of camphor Soot: Spectral analysis and structural information," Int. J. Electrochem. Sci., vol. 8, no. 7, pp. 94219428, 2013.

[16] O. Beyssac, B. Goffé, J. P. Petitet, E. Froigneux, M. Moreau, and J. N. Rouzaud, "On the characterization of disordered and heterogeneous carbonaceous materials by Raman spectroscopy," Spectrochim. Acta - Part A Mol. Biomol. Spectrosc., vol. 59, no. 10, pp. 22672276, 2003.

[17] K. Ishimaru, T. Hata, P. Bronsveld, T. Nishizawa, and Y. Imamura, "Characterization of sp2- and sp3-bonded carbon in wood charcoal," J. Wood Sci., vol. 53, no. 5, pp. 442-448, 2007.

[18] J. Henriksson, "Raman spectroscopy for characterization of soot and other carbonaceous materials," LUP Student Pap. no 8979551, no. May, 2019.

[19] M. Kawakami, T. Karato, T. Takenaka, and S. Yokoyama, "Structure analysis of coke, wood charcoal and bamboo charcoal by Raman spectroscopy and their reaction rate with CO2," ISIJ Int., vol. 45, no. 7, pp. 1027-1034, 2005.

[20] C. Karunakaran and L. G. Tabil, "Potential Applications of Infrared and Raman Spectromicroscopy for Agricultural Biomass," Agric. Eng. Int. CIGR J., vol. 0, no. 0, 2009.

[21] N. B. Yong Sik Ok, Sophie M. Uchimiya, Scott X. Chang, Biochar: Production, Characterization, and Applications. 2015.

[22] D. B. Wiedemeier et al., "Aromaticity and degree of aromatic condensation of char," Org. Geochem., vol. 78, pp. 135-143, 2015.

[23] J. R. Ferraro, K. Nakamoto, and C. W. Brown, Introductory Raman Spectroscopy Second Edition. 2003.

[24] C. N. Banwell and E. M. McCash, "Fundamentals of Molecular Spectroscopy: 4e." 1996.

[25] H. Johansson, "Characterization of biomass pyrolysis and gasification using Raman Spectroscopy," LUP Student Pap. no 8951168, no. May, 2018.

[26] R. Krishna, T. J. Unsworth, and R. Edge, Raman Spectroscopy and Microscopy, no. December. Elsevier Ltd., 2016.

[27] A. C. Ferrari, "Raman spectroscopy of graphene and graphite: Disorder, electron-phonon coupling, doping and nonadiabatic effects," Solid State Commun., vol. 143, no. 1-2, pp. 47$57,2007$. 
[28] A. C. Ferrari et al., "Raman spectrum of graphene and graphene layers," Phys. Rev. Lett., vol. 97, no. 18, pp. 1-4, 2006.

[29] N. Larouche and B. L. Stansfield, "Classifying nanostructured carbons using graphitic indices derived from Raman spectra," Carbon N. Y., vol. 48, no. 3, pp. 620-629, 2010.

[30] M. Knauer, M. Carrara, D. Rothe, R. Niessner, and N. P. Ivleva, "Changes in structure and reactivity of soot during oxidation and gasification by oxygen, studied by micro-Raman spectroscopy and temperature programmed oxidation," Aerosol Sci. Technol., vol. 43, no. 1, pp. 1-8, 2009.

[31] A. Sadezky, H. Muckenhuber, H. Grothe, R. Niessner, and U. Pöschl, "Raman microspectroscopy of soot and related carbonaceous materials: Spectral analysis and structural information," Carbon N. Y., vol. 43, no. 8, pp. 1731-1742, 2005.

[32] C. Guizani, K. Haddad, L. Limousy, and M. Jeguirim, "New insights on the structural evolution of biomass char upon pyrolysis as revealed by the Raman spectroscopy and elemental analysis," Carbon N. Y., vol. 119, pp. 519-521, 2017.

[33] D. M. Keown, J. I. Hayashi, and C. Z. Li, "Drastic changes in biomass char structure and reactivity upon contact with steam," Fuel, vol. 87, no. 7, pp. 1127-1132, 2008.

[34] Alex C. Wiedenhoeft, "Structure and Function of Wood," in Handbook of Wood Chemistry and Wood Composites, 2013. 